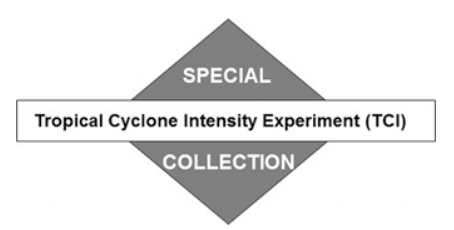

\title{
The Unexpected Rapid Intensification of Tropical Cyclones in Moderate Vertical Wind Shear. Part III: Outflow-Environment Interaction
}

\author{
DAVID R. Ryglicki, JAmes D. DOYLE, AND DANIEL HodysS \\ Naval Research Laboratory, Monterey, California \\ JoshuA H. CossuTH \\ Naval Research Laboratory, Washington, D.C. \\ Yi Jin, KeVIN C. Viner, AND Jerome M. SCHMidT \\ Naval Research Laboratory, Monterey, California
}

(Manuscript received 23 October 2018, in final form 31 May 2019)

\begin{abstract}
Interactions between the upper-level outflow of a sheared, rapidly intensifying tropical cyclone (TC) and the background environmental flow in an idealized model are presented. The most important finding is that the divergent outflow from convection localized by the tilt of the vortex serves to divert the background environmental flow around the TC, thus reducing the local vertical wind shear. We show that this effect can be understood from basic theoretical arguments related to Bernoulli flow around an obstacle. In the simulation discussed, the environmental flow diversion by the outflow is limited to $2 \mathrm{~km}$ below the tropopause in the $12-14-\mathrm{km}(250-150 \mathrm{hPa})$ layer. Synthetic water vapor satellite imagery confirms the presence of upshear arcs in the cloud field, matching satellite observations. These arcs, which exist in the same layer as the outflow, are caused by slow-moving wave features and serve as visual markers of the outflow-environment interface. The blocking effect where the outflow and the environmental winds meet creates a dynamic high pressure whose pressure gradient extends nearly $1000 \mathrm{~km}$ upwind, thus causing the environmental winds to slow down, to converge, and to sink. We discuss these results with respect to the first part of this three-part study, and apply them to another atypical rapid intensification hurricane: Matthew (2016).
\end{abstract}

\section{Introduction}

Unlike the lower- and midlevel dynamics of a tropical cyclone (TC), a topic that has appeared frequently in the literature for nearly 60 years (e.g., Palmén and Riehl 1957), the upper levels and the outflow of TCs have not been as active an area of study until very recently because of insufficient upper-level observations (Jordan 1952). With the advent of satellite and satellite-track winds, now more commonly known as atmospheric motion vectors (AMVs; Velden et al. 1997), the upper levels of the TC were able to be analyzed more carefully. Black and Anthes (1971) were able to describe, both quantitatively and qualitatively, the flow around five TCs out to a radius of $1000 \mathrm{~km}$ from the storm center.

Corresponding author: David R. Ryglicki, david.ryglicki@ nrlmry.navy.mil
They demonstrated, via Fourier analyses, that the outflow layer of an observed TC is very asymmetric. Qualitatively, they also showed that outflow is favored in jets emanating from the core of the storm. Merrill (1988a) provided a significant step forward in the understanding of TC outflow by compositing the upperlevel environment of $37 \mathrm{TCs}$. He found that upper-level TC structures can be grouped into four categories, each with its own outflow jet, and he also indicated that the upper-level environment could play a significant role in assisting these outflow jets or forming additional ones.

There has been a renewed interest in the outflow layer of a TC, as a recent field campaign, Tropical Cyclone Intensification (TCI), has focused on the evolution of TC outflow (Doyle et al. 2017). Emanuel and Rotunno (2011) and Emanuel (2012) have hypothesized that TC outflow stratifies itself based on inflow characteristics. This stratification is based on the outflow potential 
temperature profiles relative to a critical Richardson number and can be related to the intensity of the TC.

Several studies have attempted to relate outflow behavior to intensification rates. In a series of papers, Molinari and Vollaro (1989, 1990), Molinari et al. (1995), Hanley et al. (2001), and Ditchek et al. (2017) all focused on the dynamical evolution and eddy fluxes at upper levels of TCs in their respective outflow layers. Their work indicates that given a sufficiently large eddyflux convergence aloft, the TC can intensify (DeMaria et al. 1993). Specifically, the work by Hanley et al. (2001) indicates that there are differences between favorable and unfavorable locations for TC intensification, as the proximity of a TC to a passing upper-level trough may play a key role in the intensification of a TC by influencing its outflow. Schematically, Merrill (1988a) observed only one outflow jet, but a passing trough could assist a TC by helping to create a second outflow jet (Merrill and Velden 1996), thus allowing for more mass evacuation as a result of TC convection. Rappin et al. (2011) and Komaromi and Doyle (2018) both tested this hypothesis directly and found that a passing trough could aid in the focusing of an outflow channel, which in turn aids in the development of a simulated TC. Various other studies have attempted to quantify the extent to which an upper-level synoptic system can aid or inhibit the growth of a TC (Shi et al. 1997; Bosart et al. 2000; Persing et al. 2002; Möller and Shapiro 2002; Shapiro and Möller 2003; Davis and Bosart 2003; Rappin et al. 2011; McTaggart-Cowan et al. 2013).

As Hanley et al. (2001) discussed, and a fact to which many previous works allude, there are noticeable differences among ventilating TCs, inducing a potential vorticity-related spinup, and outright shearing of the TC, depending on the proximity of the upper-level synoptic forcing. Vertical wind shear (VWS) is generally a negative influence on TC intensification (Merrill 1988b; Wang and Wu 2004). Recent studies on wind shear's negative effects have focused on the thermodynamic effects of VWS, such as the midlevel ventilation (Tang and Emanuel 2010; Tang and Emanuel 2012; Ge et al. 2013) or the flushing of the boundary layer by low moisture air (Riemer et al. 2010; Riemer and Laliberté 2015). Despite those thermodynamic effects, Onderlinde and Nolan (2016) and Finocchio et al. (2016) have demonstrated that given the correct environmental setup, the helicity of the background flow or the depth of the background winds can drastically change the evolution of a simulated TC. It is the depth of the background flow that Ryglicki et al. (2018a, hereafter Part I) have argued is instrumental for explaining why a subset of TCs overcome the prevailing negative effects of shear to not only intensify but also undergo rapid intensification when the large-scale $200-850$-hPa vertical wind shear would climatologically indicate inhibited intensification. They argued that this "atypical" pathway to rapid intensification (RI) is distinct from classical understandings of TC rapid intensification (Ooyama 1982; Rotunno and Emanuel 1987; Montgomery et al. 2009) given the evolution of the vortex tilt.

This work serves as a numerical model-based companion to Part I and as a follow-up to Ryglicki et al. (2018b, hereafter Part II). In the satellite observations presented in Part I, they noted two key identifying features: tilt-modulated convective asymmetries (TCA), which appear prior to RI and with a period of 4-8 h and upper-level arcs, which appear in the water vapor (WV) satellite imagery. Part II demonstrated that these TCAs are associated with the nutation ${ }^{1}$ of the tilt of the vortex. The analysis of Part II focused on the tilt and the structure of the TCAs. This work will focus on some of the effects that the TCAs have on the TC and its environment. Part I alluded to the fact that the outflow serves to divert the upper-level flow ("E" from Fig. 1 of Part I). This study focuses on the blocking characteristics, including why the depth of the environmental flow is so important and how the environment is in turn affected by the block.

Section 2 provides an overview of the simulations, general diagnostics of the simulations, and the TC center-finding methods. Section 3 presents the physical characteristics of the outflow and also explores the outflow-environment boundary and the far-field response. Section 4 relates the findings back to observations from Part I in addition to presenting an example of how this analysis would be useful in an operational setting. Section 5 provides a summary of the findings herein, synthesizes the results and discussions of this series, and presents ideas for future work.

\section{Experiment description, general diagnostics, and methodology}

\section{a. Experiment description}

The model used for this work is a slightly modified Cloud Model 1 (CM1; Bryan and Fritsch 2002) release 18. Full details of the simulations can be found in Part II. A thermally balanced background is specified for a prescribed environmental flow. Four simulations are run: three sheared and one nonsheared control. The thermodynamic base state is the Dunion "moist tropical" sounding (Dunion 2011). The tropopause

\footnotetext{
${ }^{1}$ A periodic variation in the inclination of the axis of a rotating object (i.e., the vortex).
} 

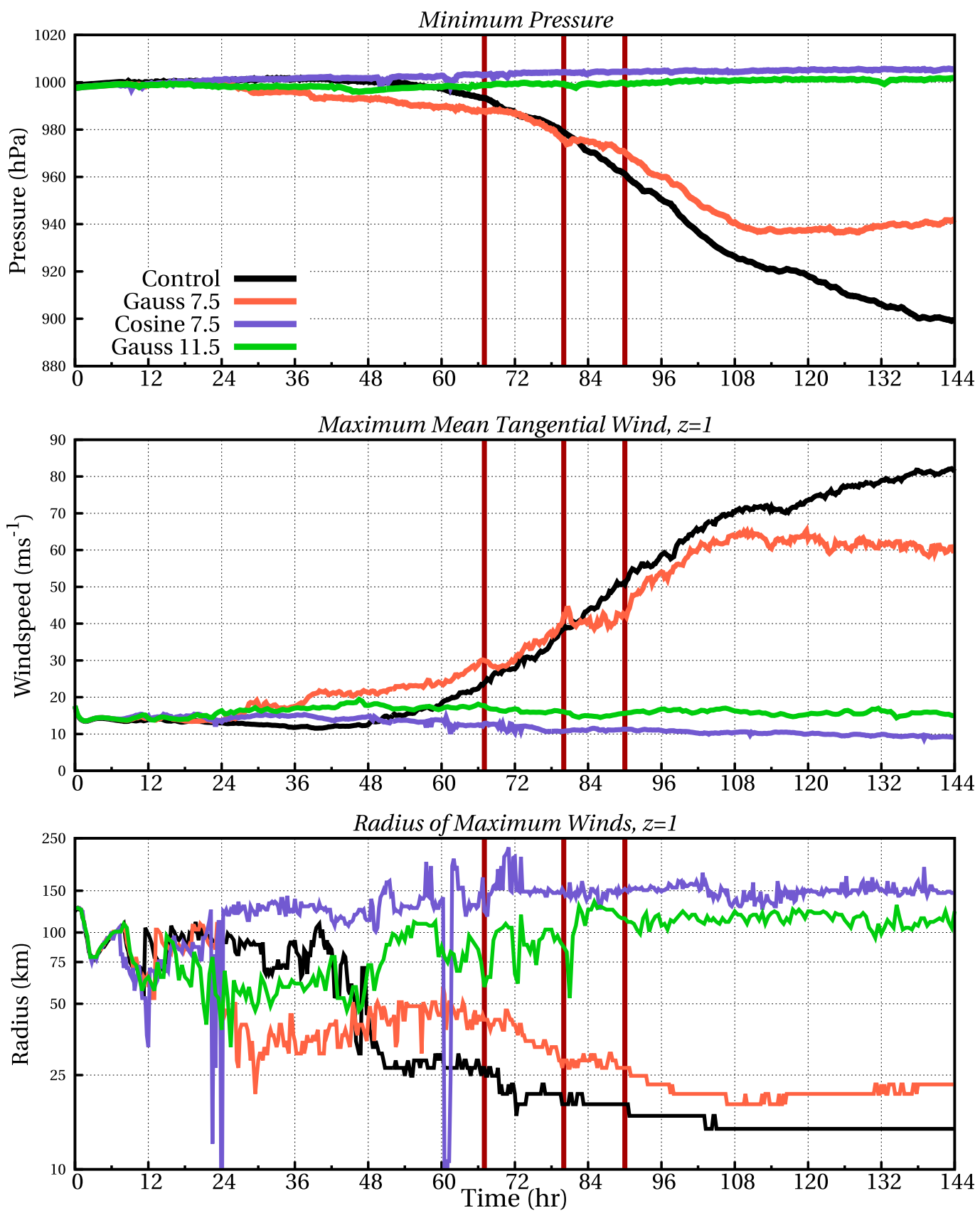

FIG. 1. (top) Minimum pressure, (middle) maximum mean tangential wind, and (bottom) radius of maximum winds, all at the lowest scalar model level, for the four simulations. Dark red lines correspond to the first rapid intensification period, the levelling off of intensification, and the second abrupt rapid intensification of the Gaussian-7.5 TC.

$\left(N^{2}=2 \times 10^{-4} \mathrm{~s}^{-1}\right)$ is at $14 \mathrm{~km}$. SSTs are horizontally homogenous and invariant in time with a value of $27.5^{\circ} \mathrm{C}$ (Part I). An $f$ plane at $20^{\circ} \mathrm{N}$ is used. Three background wind profiles are used: a Gaussian profile with a $13-1.5-\mathrm{km}$ (proxy for $200-850 \mathrm{hPa}$ ) shear value of $7.5 \mathrm{~m} \mathrm{~s}^{-1}$ (hence, G7.5), a cosine profile with a shear value also of $7.5 \mathrm{~m} \mathrm{~s}^{-1}(\mathrm{C} 7.5)$, and an additional
Gaussian profile with a shear value of $11.5 \mathrm{~m} \mathrm{~s}^{-1}$ (G11.5). The background environmental winds for the control are zero everywhere. As a reminder, $7.5 \mathrm{~m} \mathrm{~s}^{-1}$ is the average shear value from the six TCs in Part I. The two profiles are meant to mimic, in an idealized sense, the depth of the flow from an upper-level anticyclone (Gaussian) and an upper-level trough (cosine). Please 
refer to Part II for more details, although we would note that the TCs are initialized only up to $10-\mathrm{km}$ height.

\section{b. General diagnostics}

Aspects of the simulations have been discussed in Part II; however, this section will provide a brief review of the results particularly relevant for this study. Figure 1 (reproduced from Part II) illustrates the minimum pressure, maximum mean tangential wind, and RMW of the four simulations from Part II. As noted in Part II, the control develops, the G7.5 TC develops, the C7.5 TC does not develop, and the G11.5 TC does not develop.

\section{c. Center-finding and shear-calculating methodology}

As in Part II, multiple center-finding methods were used. Table 1 documents all of the center-finding methods discussed in this manuscript. They are a tiltrelative wind method that seeks to maximize the mean tangential wind at the RMW (MAVWBS), a tilt-relative PV-vorticity hybrid centroid (PVV120), and a stormrelative (surface-centered) minimum of a smoothed pressure field (PMIN-SM9). See Part II for more description.

In section 3, a localized, storm-relative (i.e., centered on the low-level pressure minimum) vertical wind shear is computed. We average over the two closest levels to 1.5 and $13.0 \mathrm{~km}$. The zonal and meridional components are averaged individually before creating a wind vector for a given layer from the mean components. This effectively removes the mean vortex circulation. Each layer is then given a mean wind magnitude, and the shear magnitude is the subtraction of the lower layer from the upper layer. This shear measure is used as a diagnostic in section 3 .

\section{d. AMV analysis}

In section 4, we analyze AMV data directly for a select case. AMVs, provided on a 3-hourly basis by the Cooperative Institute for Meteorological Satellite Studies (CIMSS), are interpolated to a storm-centered grid using a Cressman-style (Cressman 1959) analysis. For simplicity, all AMVs at $250 \mathrm{hPa}$ and higher are treated as being on the same vertical level. The AMVs are horizontally interpolated using inverse quadratic radial basis functions:

$$
\phi(d)=\frac{1}{1+(d / \varepsilon)^{2}},
$$

where $d$ is the great circle distance between a grid point and an observation, and $\varepsilon$ is a weighting factor. After trial and error, $\varepsilon$ is set to $50 \mathrm{~km}$ in order to smooth the wind field, thus preserving features with scales of
TABLE 1. All of the center-finding methods used in this paper, including their classes (Ryglicki and Hart 2015): minimization of azimuthal variance (MAV), local extreme (LE), and weighted grid points (WGP).

\begin{tabular}{|c|c|c|}
\hline Abbreviation & Class & Description \\
\hline MAVWBS & MAV & $\begin{array}{l}\text { Brute force check for maximum mean } \\
\text { tangential wind at each grid point in } \\
100-\mathrm{km} \text { box around first guess out to a } \\
\text { radius of } 150 \mathrm{~km} \text {, then Hurricane } \\
\text { Research Division simplex (Marks et al. } \\
\text { 1992). Annulus size of } 4 \sqrt{ } 2 \mathrm{~km} \text { (inner- } \\
\text { domain } d x \text { multiplied by } 2 \sqrt{ } 2 \text { ) }\end{array}$ \\
\hline PMIN-SM9 & LE & Minimum of the smoothed pressure field \\
\hline PVV120 & WGP & $\begin{array}{l}\text { Blended vorticity and potential vorticity } \\
\text { centroids, } 120 \mathrm{~km} \times 120 \mathrm{~km} \text { weighting } \\
\text { box: vorticity centroid up to } 500 \mathrm{~m} \text {, } \\
\text { Hermitian blend to } 4 \mathrm{~km} \text {, potential } \\
\text { vorticity centroid to top of storm }\end{array}$ \\
\hline
\end{tabular}

20-200 km. Only the nearest 20 observations to a grid point are used. All AMVs are transformed relative to the TC center as per the method of Ahern and Cowan (2018) to account for the curvature of Earth.

\section{Upper-level model results}

\section{a. Conceptual overview}

A schematic, summarizing the findings of this manuscript, of the entire outflow-environment complex in the G7.5 simulation is shown in Fig. 2. We would remind the reader that this depiction is only a two-dimensional, top-down perspective of the physical processes occurring at approximately $200-\mathrm{hPa}$ or $12.5-13-\mathrm{km}$ height. This setup is not what is normally expected of outflow based on previous studies (e.g., Merrill 1988a; Merrill and Velden 1996; Sears and Velden 2014), where the outflow of a TC undergoing RI is expected to expand in practically all radial directions. The primary assumption in Fig. 2 is that the strongest winds of the outflow are aligned vertically in opposition to the strongest winds of the environment. In addition, Fig. 2 depicts outflow's being expelled during a TCA, so this upper-level structure forms prior to vertical vortex alignment and RI.

There are three primary upshear regions of interest (I, II, and III), and two upshear radii of interest (A and B). Starting from the TC, (I) is TC outflow. Outflow is simply characterized as air that is ejected from the convection in the TC. Initially, it can be characterized as primarily divergent flow. The first boundary (A), which is henceforth referred to as the "outflow front," is where the outflow and the environment meet. The outflow front is delineated by a density gradient, a reversal of the radial flow, a large $(>40 \%)$ decrease in relative 


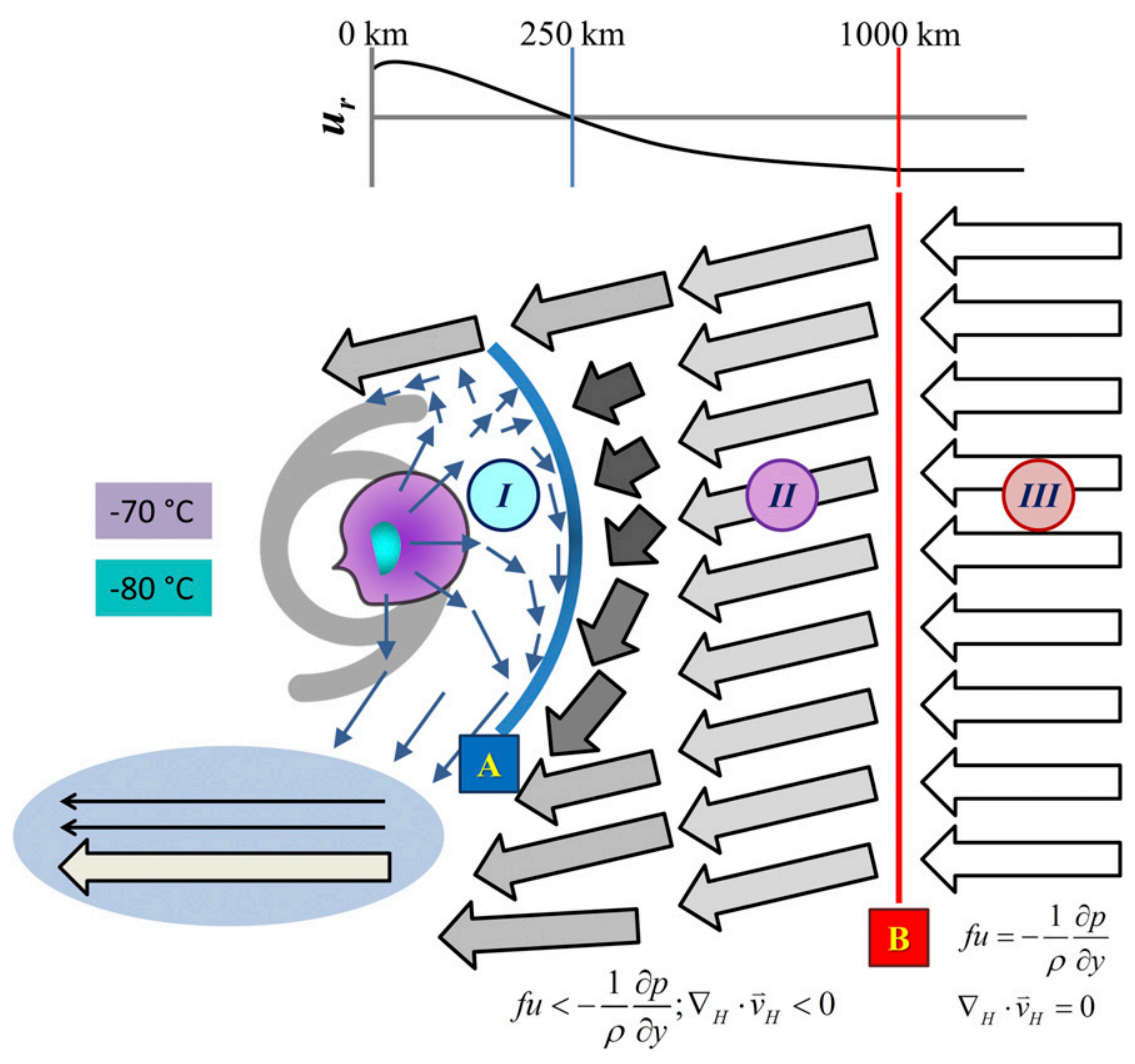

FIG. 2. Schematic illustrating the outflow of a TC that distorts the environmental winds. Large thick arrows are environmental flow; thin arrows are TC outflow. Outline of lavender and teal colors indicates satellite imagery. The blue-shaded oval is the outflow jet. The letters (A and B) and roman numerals (I, II, and III) indicate the various boundaries and regions, respectively. Region I is strictly the outflow, and it is defined as having density lower than that of the surrounding atmosphere at a given level. A is the outflow front, where the outflow and the environment collide. Beyond the outflow front lies Region II, where the environmental winds become subgeostrophic, decelerate, converge, slowly sink, and turn to the left. B indicates the bow wave, which is the farthest boundary where the outflow affects the environmental winds. Region III is the unaffected free atmosphere in geostrophic thermal wind balance. Spatially, radially from the core, A exists approximately 200 to $250 \mathrm{~km}$ upshear and B exists approximately $1000 \mathrm{~km}$ upshear. Top line is radial wind at different radii. Shear vector is coincident with environmental winds (easterly).

humidity, and a deformation line (cf. Bluestein 1992, p. 239). This front typically exists $200-300 \mathrm{~km}$ radially outward upshear of the low-level center. This front can be traced left-of-shear, where it eventually ends in an outflow jet (blue oval) as the outflow and the environmental flow merge together.

Beyond the front lies a large area $(250-1000 \mathrm{~km}$ radially outward) of convergence where the environmental flow is slowed down by ancillary effects of the block (II; between A and B). In this region, our analysis shows that the wind is subgeostrophic, and the environmental flow both curves to the left and slowly descends, acknowledging the limitations of geostrophic balance in the tropics. A bow wave is located $\sim 1000 \mathrm{~km}$ beyond the outflow front. It represents the farthest extent of the TC where environmental winds return to geostrophic balance.

As with the entire "atypical" RI process (Part I), this is a complex physical structure. As a result, we are going to perform a detailed analysis of the key physical components: the outflow itself (region I) and the regions (II and III) beyond the outflow front. Section $3 b$ focuses on boundary A (the outflow front) and how Regions I and II interact around the boundary; section $3 c$ examines Regions II and III.

\section{b. Outflow-environment interface}

Asymmetric tilt-modulated convection dominates the evolution of the G7.5 TC until the second RI period at $90 \mathrm{~h}$ (Part II). This convection serves an important role 
(a) $000 \mathrm{hr} 00 \mathrm{~min}$

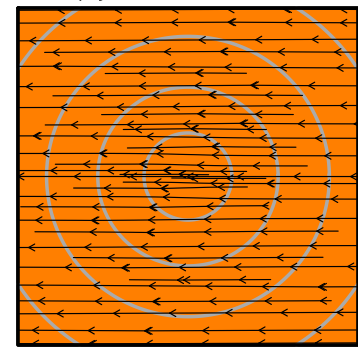

(d) $035 \mathrm{hr} 00 \mathrm{~min}$

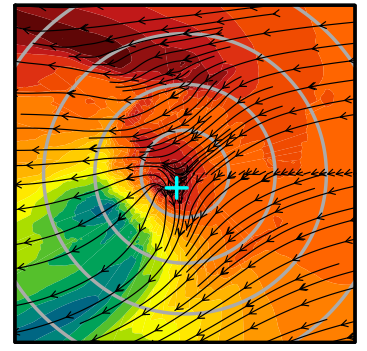

(g) $053 \mathrm{hr} 15 \mathrm{~min}$

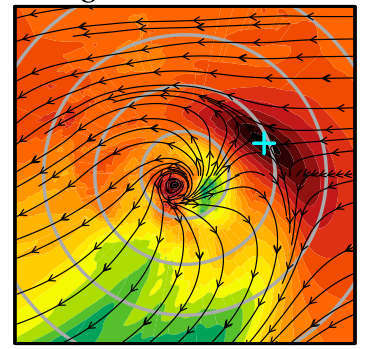

(b) $016 \mathrm{hr} 15 \mathrm{~min}$

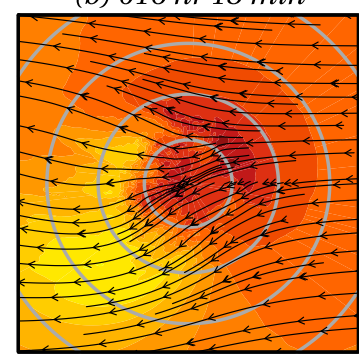

(e) $040 \mathrm{hr} 00 \mathrm{~min}$

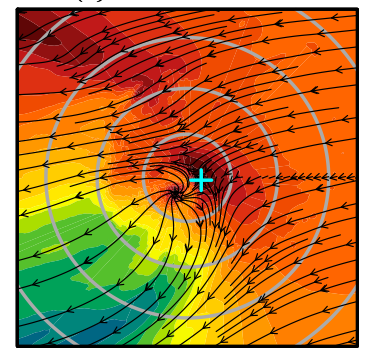

(h) $062 \mathrm{hr} 15 \mathrm{~min}$

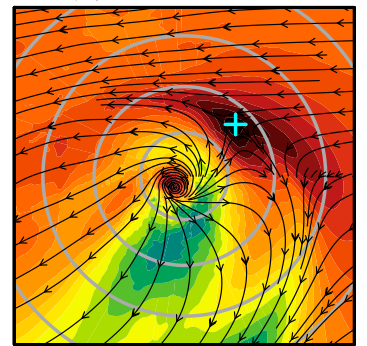

(c) $028 \mathrm{hr} 00 \mathrm{~min}$

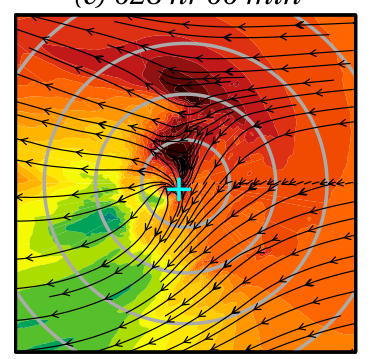

(f) $044 \mathrm{hr} 45 \mathrm{~min}$

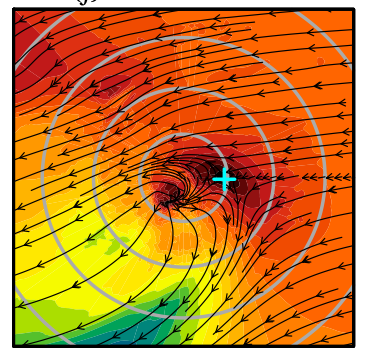

(i) $068 \mathrm{hr} 15 \mathrm{~min}$

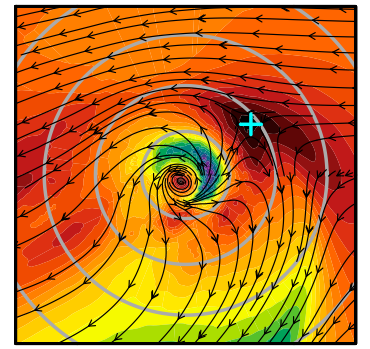

FIG. 3. Streamlines of the storm-relative $12.6-\mathrm{km}$ winds $\left(\mathrm{m} \mathrm{s}^{-1}\right)$, smoothed with a 2.5 -h running mean and colored by magnitude $\left(\mathrm{m} \mathrm{s}^{-1}\right.$ ), (a) at initial time, (b) $16 \mathrm{~h} 15 \mathrm{~min}$, (c) $28 \mathrm{~h} 00 \mathrm{~min}$, (d) $35 \mathrm{~h} 00 \mathrm{~min}$, (e) $40 \mathrm{~h} 00 \mathrm{~min}$, (f) $44 \mathrm{~h}$ $45 \mathrm{~min}$, (g) $53 \mathrm{~h} 15 \mathrm{~min}$, (h) $62 \mathrm{~h} 15 \mathrm{~min}$, and (i) $68 \mathrm{~h} 15 \mathrm{~min}$. Gray circles are 100-km intervals. Cyan cross is the stagnation point (winds approach zero at outflow front).

at the upper levels of the TC. Figure 3 is a collection of snapshots from the G7.5 simulation of smoothed streamlines of the storm-relative winds at $12.6 \mathrm{~km}$. These images indicate that the outflow from the convection of the TC is creating a region that diverts the environmental wind. The model TC is only initialized up to $10 \mathrm{~km}$ in the vertical (Fig. 3a); therefore, convection and the secondary circulation is required to further develop the $\mathrm{TC}$ in the vertical and divert the environmental flow (Fig. 3b). Following Bernoulli flow theory, we introduce a concept from this literature: the stagnation point, which is defined as where the winds reduce to zero. As shown throughout Fig. 3, the stagnation point (cyan-colored cross) is steadily pushed upwind beginning downshear-left (Figs. 3c,f) as the TC builds itself. This is not a constant forcing, however, as the minimum moves outward to $200 \mathrm{~km}$ (Fig. 3g), moves inward to $150 \mathrm{~km}$ (Fig. 3h), and then returns to $200 \mathrm{~km}$ (Fig. 3i). To quantify this behavior in time, the outflow front is henceforth defined as the radius at which the radial wind, averaged from $-30^{\circ}$ to $30^{\circ}$ upshear, changes sign.

Figure 4 presents the 6-km tilt angle, convection, 6-km tilt magnitude, and upshear radial winds at $12.6-\mathrm{km}$ height. We switch to a tilt-relative perspective for the radial winds to account for the tilt movement and to identify local effects at upper levels. The 6-km tilt is used to document the location of the midlevel center (see Part II). Convection is quantified by the total condensed water in the column (TCWC; see Part II for definition). The upshear outflow front, marked by the radial wind zero line, initially only extends to $50 \mathrm{~km}$ for the first $42 \mathrm{~h}$ (Fig. 4c). From 42 to $54 \mathrm{~h}$, as the tilt angle moves counterclockwise, or cyclonically, $45^{\circ}$ (Fig. 4a), the outflow pushes the environmental winds back considerably, from 50 to $200 \mathrm{~km}$ in $12 \mathrm{~h}$ (Fig. 4c). We would note here that it is during this time frame that the precession component of the tilt reaches left-of-shear (see Part II). 

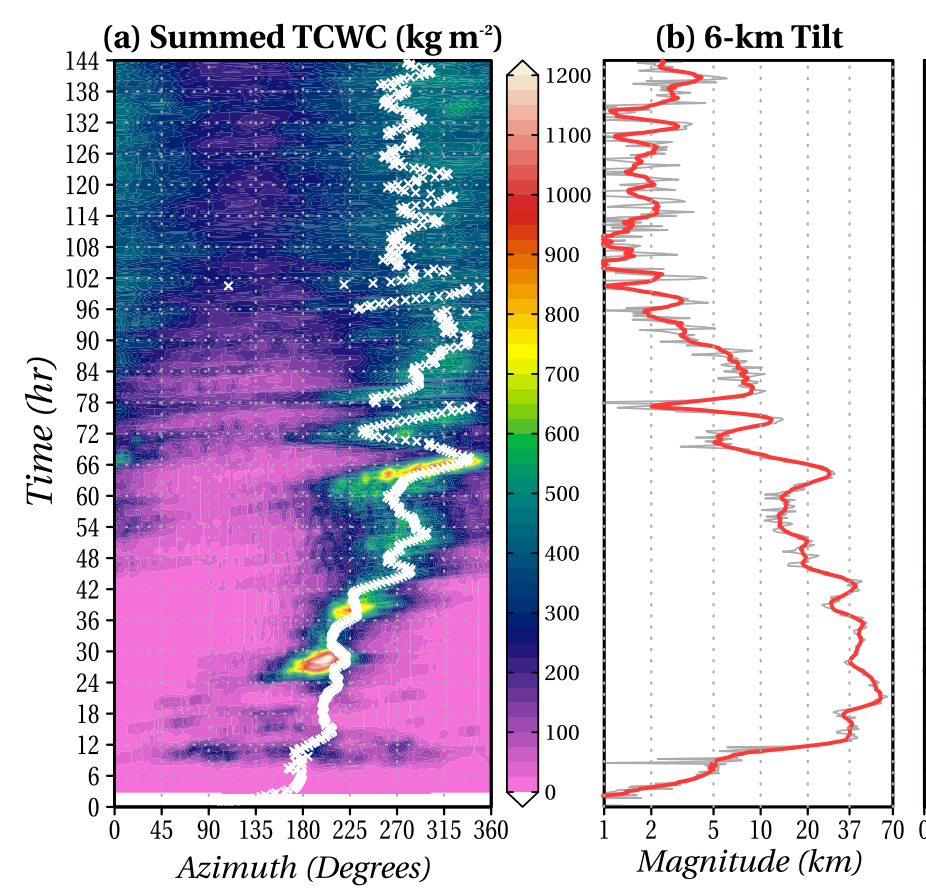

(c) $12.6 \mathrm{~km} u_{r}\left(\mathrm{~ms}^{-1}\right)$

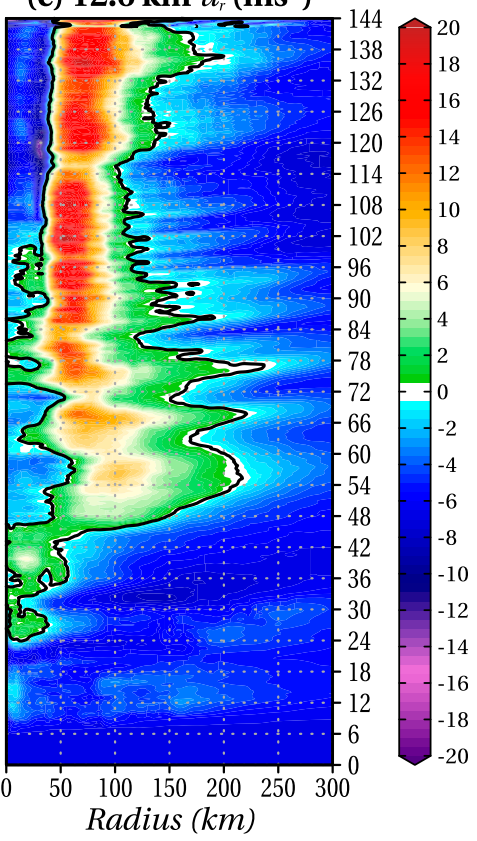

FIG. 4. Storm-relative total condensed water of the column (TCWC, $\mathrm{kg} \mathrm{m}^{-2}$ ), (a) radially summed from 0 to $100 \mathrm{~km}$, with the 6-km tilt angle overlaid (white crosses), where the tilt is calculated using the potential vorticity hybrid method; (b) the 6-km tilt magnitude; and (c) the tilt-relative upshear radial wind ( $\mathrm{m} \mathrm{s}^{-1}$ ), with zero contoured in black, at $12.6-\mathrm{km}$ height. All fields are smoothed with a 2.5 -h running mean except the gray line in (b). In (a), azimuthal angles are math convention, where $0^{\circ}$ is east (upshear), $90^{\circ}$ is north, and so on.

The front then oscillates with the strongest convection between 150 and $250 \mathrm{~km}$ upshear of the $12.6-\mathrm{km}$ center. The outflow emanates from the convection and interacts with the upper-level winds. Conceptually, this matches with discussions by Black et al. (2002) and Eastin et al. (2005) where the convection initiates downshear leftor, more accurately in this case, down-tilt, as identified by Jones (1995)—rotates cyclonically with the mean storm motion, and the resultant outflow exits the storm on the upshear side.

The extent of the outflow can also be identified using synthetic satellite observations. To calculate simulated WV brightness temperatures, the CM1 output was passed to the Community Radiative Transfer Model (CRTM; Van Delst 2013; Grasso et al. 2008; Bikos et al. 2012; Jin et al. 2014). Given the relatively unrealistic clear-sky radiation properties of the idealized simulation, we are only going to focus on qualitative similarities between satellite observations and the model results. In Part I, we documented very thin arc-like features on the upshear side of the TCs which underwent RI in the synthetic WV imagery. Figure 5 illustrates that similar features appear here as well. Hernan (Fig. 5a), Joaquin (Fig. 5b), and Hilda (Fig. 5c), all display very thin arcs (identified as the red $\times$ in the figures) whose brightness temperatures are between $-40^{\circ}$ and $-60^{\circ} \mathrm{C}$ on their upshear sides with a thin warm region separating them from the main cirrus shield. This is also present in the G7.5 simulation (Fig. 5d). In contrast, the control simulation (Fig. 5f) demonstrates that the cloud shield (and outflow) expands in all directions, and temperature gradients in outer clouds are more diffuse, which is similar to the "classic RI" case in light shear (Rick, Fig. 5e).

One of the contributing elements to synthetic brightness temperature is number concentration of scattering particles (ice, graupel, snow). To gain an understanding of the vertical structure of the clouds, Fig. 6a presents a 2D perspective of the number concentration of all ice particles at $12.6-\mathrm{km}$ height at $62 \mathrm{~h}$ into the simulation (as in Fig. 5d). There is a gap in the nuclei field on the eastern side of the TC between the arc and the main cloud shield, which indicates why the CRTM perceives a warm gap [Fig. 6a; the gap can be seen at coordinate $(500.0,-100.0)]$. A vertical cross section shows that this arc is due to the number concentration of ice particles between 12.5and $14.0-\mathrm{km}$ height [Fig. 6b; the gap can be seen here at $(500,13)]$, or just under the tropopause in the mean moist tropical sounding (Dunion 2011). As seen in Fig. 6b, these arcs are visual indicators approximating the outflow front. 


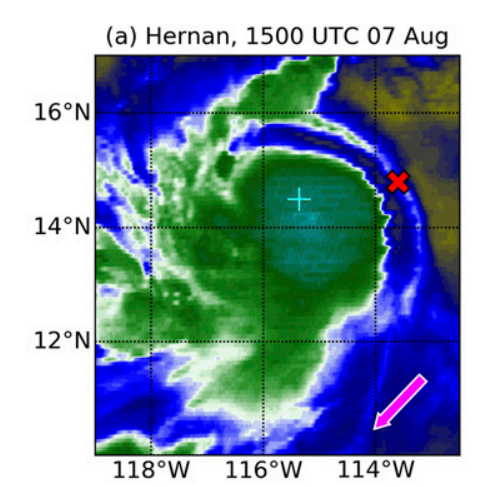

(c) Hilda, 1300 UTC 07 Aug

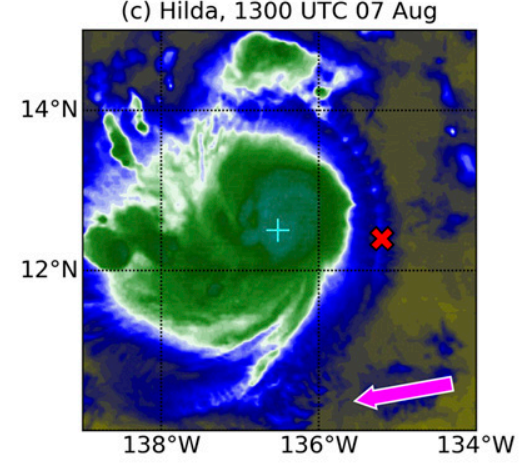

(e) Rick, 0015 UTC 17 Oct

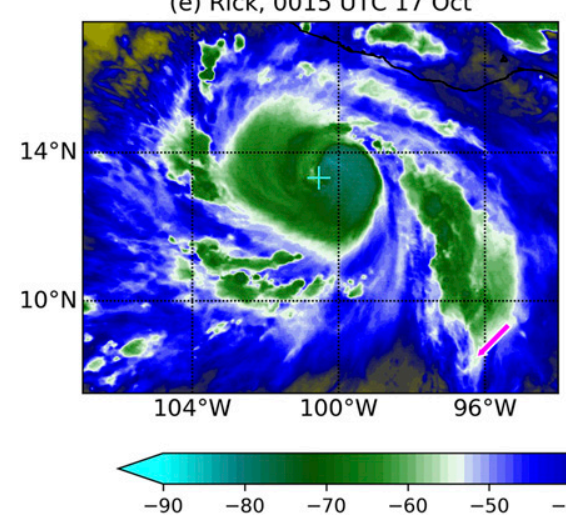

(b) Joaquin, 0315 UTC 01 Oct

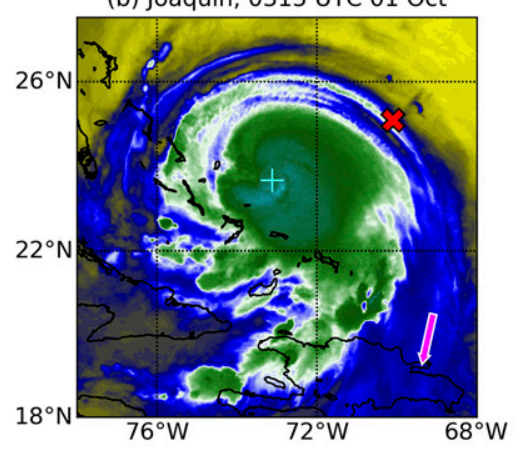

(d) CM1-shear, $62 \mathrm{hr} 00 \mathrm{~min}$

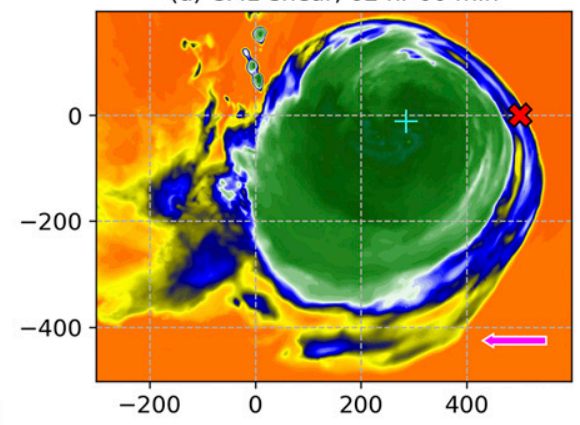

(f) $\mathrm{CM} 1$-control, $78 \mathrm{hr} 30 \mathrm{~min}$

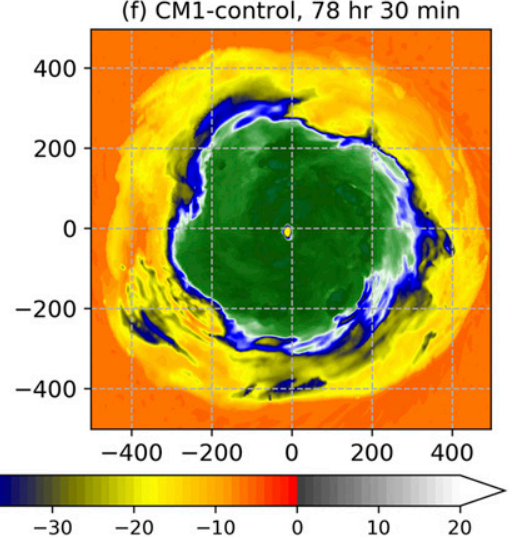

FIG. 5. Enhanced water vapor satellite imagery $\left({ }^{\circ} \mathrm{C}\right)$ for (a) 2008 Hernan, (b) 2015 Joaquin, (c) 2015 Hilda, (d) the sheared CM1 simulation (synthetic), (e) 2009 Rick, and (f) the control CM1 simulation (synthetic). Magenta arrows are shear direction only. Red " $\times$ " in (a)-(d) identifies the arc. Cyan crosses indicate either the best track center (a)-(c),(e) or the low-level center (d). Units of the abscissa and ordinates of the CM1 simulations are $\mathrm{km}$.

Physically, these arcs manifest as wavelike features passing through the outflow, and we must stress that the arcs are not the outflow front itself: they are only approximate indicators. Figure 7 presents various quantities centered on the arc at $525 \mathrm{~km}$. The winds decelerate in the outflow toward the front where there is a local pressure maximum (Fig. 7a) on the TC-side of the outflow front. The density and potential temperature fields (Fig. 7b) oscillate out of phase with each other. What the moisture variables indicate (Fig. 7c) is that the air remains near saturation before the wind changes direction. A wave-like feature of sufficiently large amplitude, centered at $500 \mathrm{~km}$ (Fig. 7b), modulates the environment sufficiently to create a warm gap in the cloud field and a local cold region where the arc is. Prior to reaching the outflow front, these features propagate with a speed of $8 \mathrm{~m} \mathrm{~s}^{-1}$ (not shown). Based on the thermal field, the radial wavelength of this feature is approximately $50 \mathrm{~km}$, which would indicate that the arc (which is due to the trough) is approximately $25 \mathrm{~km}$ in 

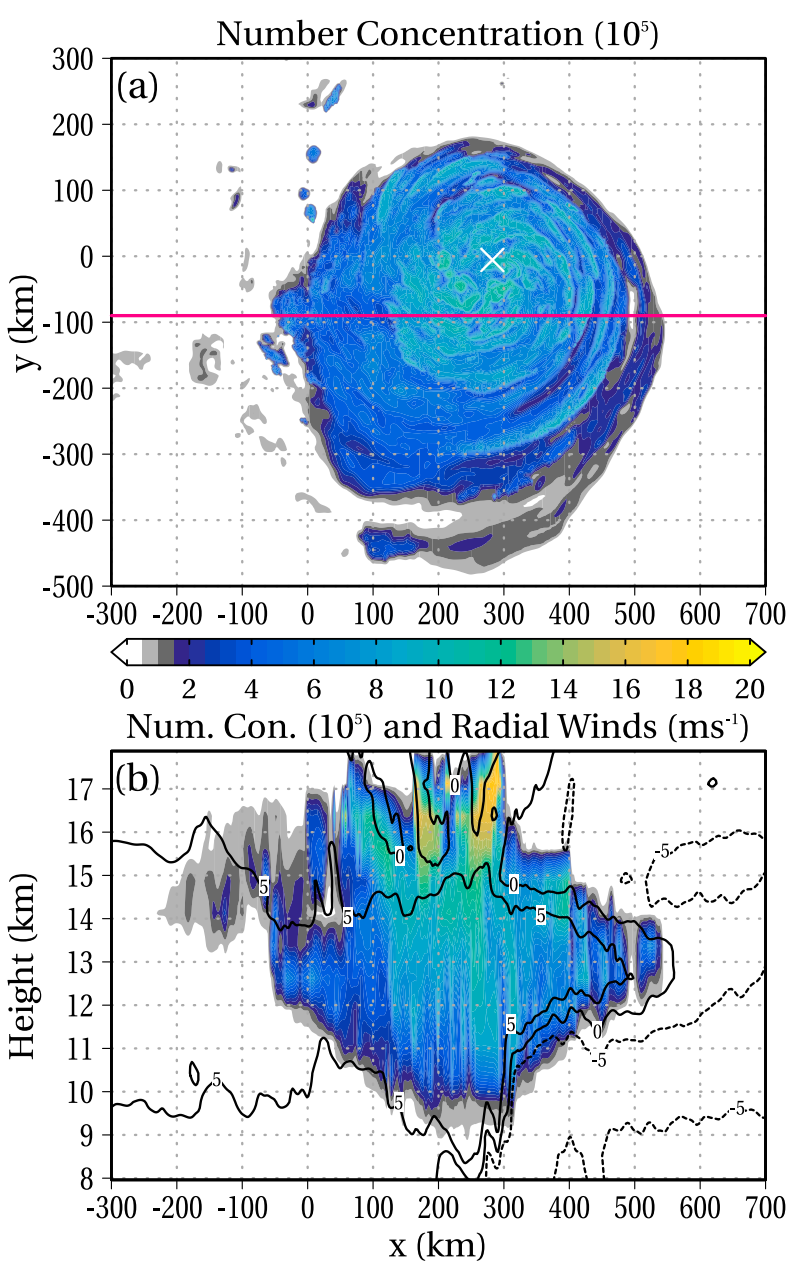

FIG. 6. (a) Number concentration of frozen condensation nuclei at $12.6-\mathrm{km}$ height at $62 \mathrm{~h}$ and (b) vertical cross section of radial winds $\left(\mathrm{m} \mathrm{s}^{-1}\right)$ and number concentration, where the magenta line in (a) indicates location of cross section. White " $\times$ " in (a) indicates low-level center.

length. Both characteristics - the wavelength and the propagation speed — are very similar to values described in Part I.

Materially, the outflow serves the purpose of reducing the shear on the vortex. From the radial wind analyses, the farthest radius that the outflow front reaches is between 200 and $250 \mathrm{~km}$. This would suggest that many other shear calculations that use annuli far outside the core or that attempt to remove the vortex may be missing a critical component of localized shear reduction. Figure 8 a illustrates this point by analyzing the shear around the low-level center in the G7.5 simulation three ways: a $0-200-\mathrm{km}$ circle, a $0-500-\mathrm{km}$ circle, and a 200-500-km annulus. The shear in the inner circle reduces from $7.5 \mathrm{~m} \mathrm{~s}^{-1}$ to approximately $3.5 \mathrm{~m} \mathrm{~s}^{-1}$. While the shear does decrease using the other two calculations, the shear decrease is much more modest using the two

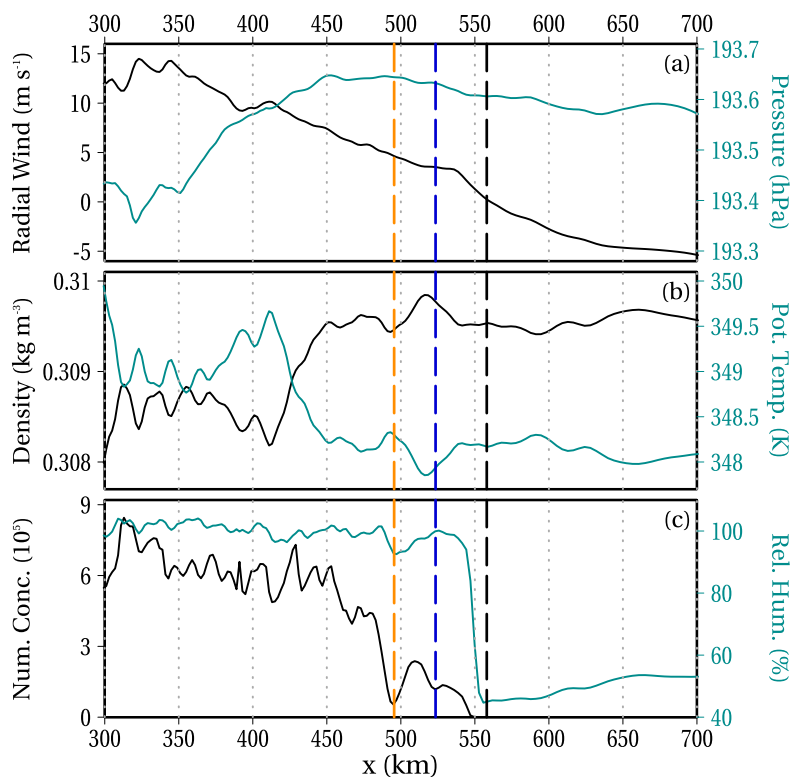

FIG. 7. (a) Radial wind and pressure, (b) density and potential temperature, and (c) number concentration of frozen condensation nuclei and relative humidity with respect to ice through the arc at $12.6 \mathrm{~km}$ identified in Fig. 7. Dashed yellow line is the gap; dashed blue line is the center of the arc; dashed black line is the outflow front (where radial winds change sign).

larger areas: $\sim 1 \mathrm{~m} \mathrm{~s}^{-1}$ reduction. When comparing the shear reduction of the three sheared TCs using the small circle (Fig. 8b), the shear is not reduced in the C7.5 simulation at all. Interestingly, there is shear reduction in the G11.5 TC, but this reduction is only, on average, $2 \mathrm{~m} \mathrm{~s}^{-1}$, which still leaves the shear value at $9.5 \mathrm{~m} \mathrm{~s}^{-1}$. In the G11.5 case, there are repeating convective events (Fig. 8c) whose cumulative effects are tied to the local shear reduction. The key difference between the two nondeveloping simulations is that in the G11.5 simulation, a midlevel structure remains coherent (not shown) but does not precess upshear. In the C7.5 simulation, the TC reduces to a low-level swirl, and any coherent midlevel structures that do form are advected away by the environment. Convection thus remains minimal (Fig. 8c).

Since the environmental flow must be rerouted, the most important interactions are taking place upshear. To analyze this, we perform a Helmholtz decomposition to compute the divergent $(\chi)$ and rotational $(\psi)$ components of the horizontal wind. We perform the wind transformation spectrally using fast Fourier transforms. Since the background winds are by definition initialized as both irrotational and nondivergent, they are completely filtered out of the resultant fields. Ignoring isolated, short-lived convection, nearly all of the rotational and divergent winds are due to the TC itself. Figure 9 is a 

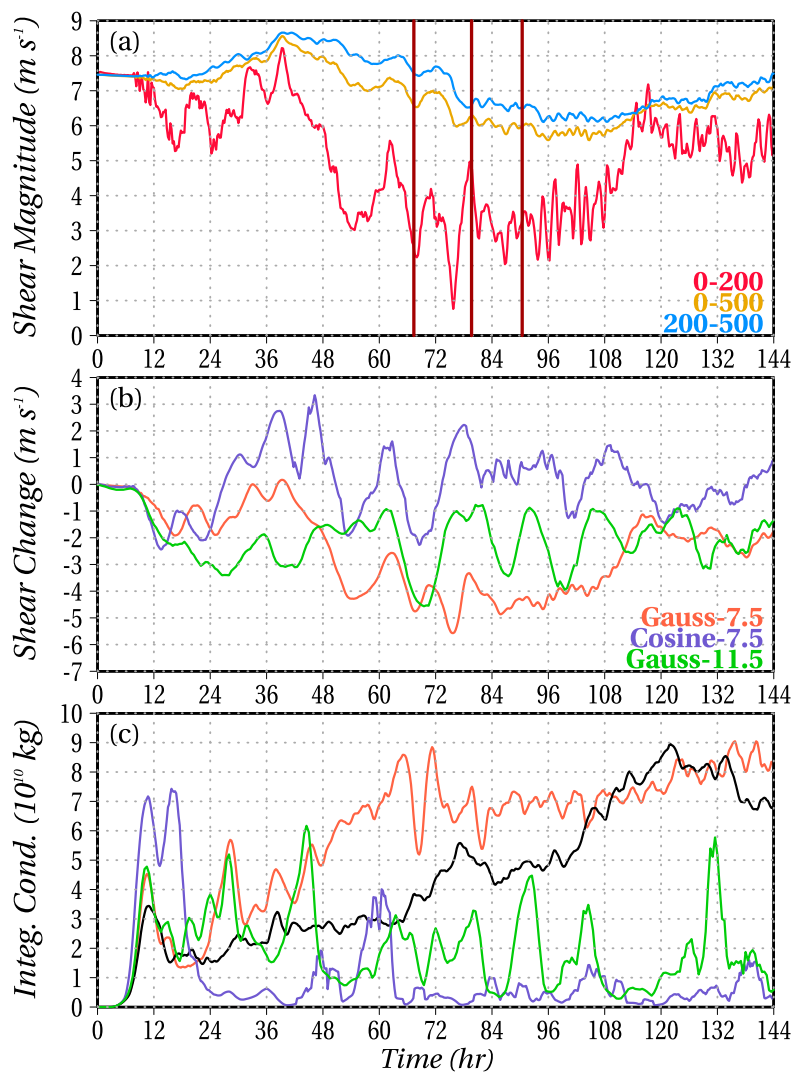

FIG. 8. (a) Storm-relative, $13-1.5-\mathrm{km}$ shear $\left(\mathrm{m} \mathrm{s}^{-1}\right)$ calculated three different ways: 0-200-km circle (red), 0-500-km circle (gold), and $200-500-\mathrm{km}$ annulus (blue) for the Gaussian-7.5 TC. Dark red lines indicate same time periods as Fig. 2. (b) Shear change (relative to initial time) using the $0-200-\mathrm{km}$ circle for each of the three sheared simulations, smoothed with a 2-h running mean. (c) Volume-integrated total condensate in a cylinder of $100-\mathrm{km}$ radius around the low-level center; black line is the control.

snapshot at $12.6 \mathrm{~km}$ of both divergent (Fig. 9a) and rotational (Fig. 9b) winds. The strongest divergent winds are focused upshear and are the main source of the blocking, while the rotational winds are deformed.

We quantify the portion of the outflow pushing back the environment as the mass-weighted divergent kinetic energy (DKE), defined thusly

$$
\mathrm{DKE}=\iiint \frac{\rho}{2}\left(u_{\chi}^{2}+v_{\chi}^{2}\right) d x d y d z .
$$

The limits of integration are the innermost $2000 \mathrm{~km}(x)$ by $1000 \mathrm{~km}(y)$ and between 9 and $16 \mathrm{~km}$ in height, ignoring inflow layers and concentrating on TC outflow. The divergent kinetic energy over the first $90 \mathrm{~h}$, which is the critical time period between genesis and the abrupt second intensification, is consistently two to three times larger in the G7.5 TC than in the control TC (Fig. 10a).
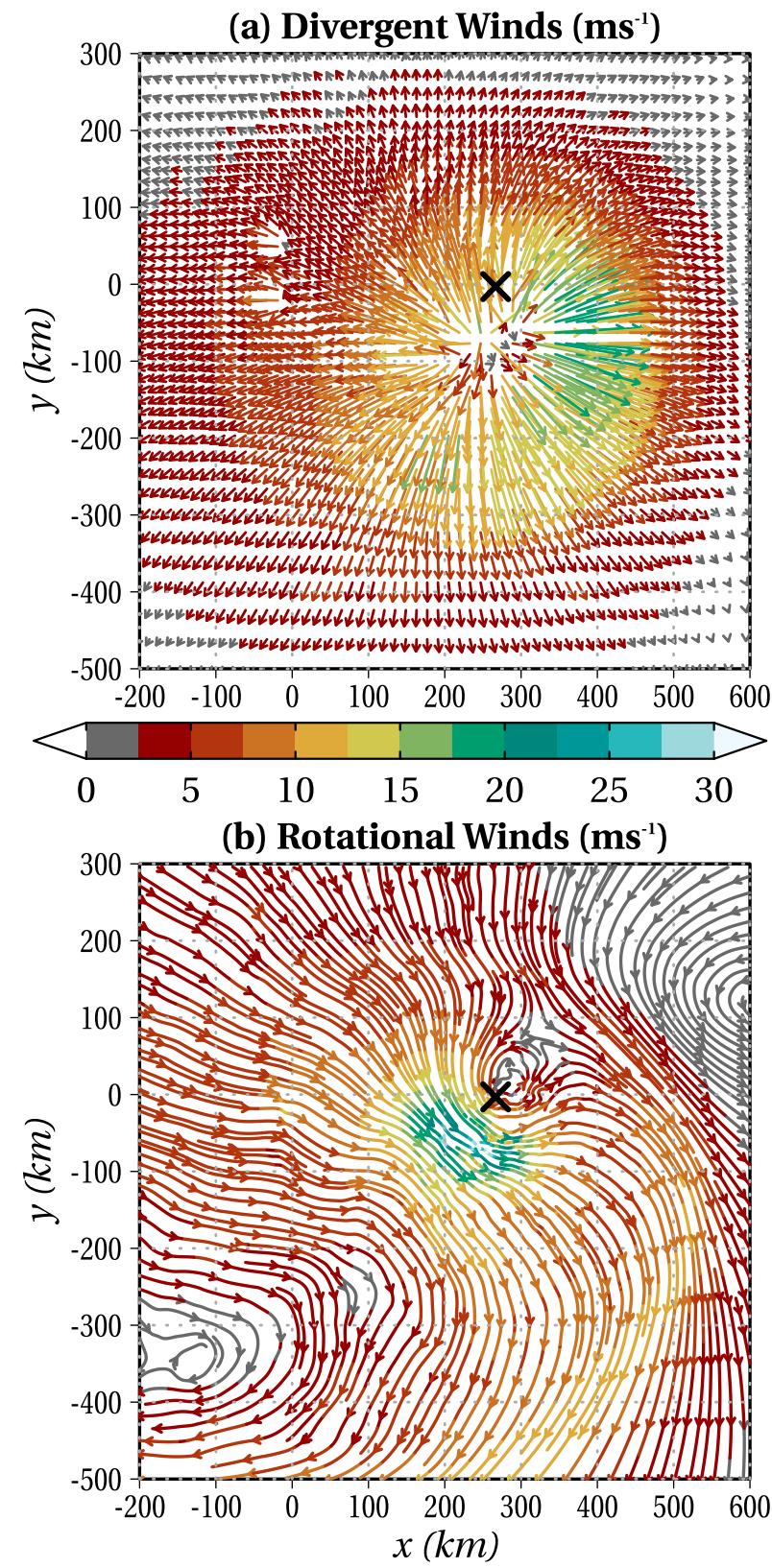

FIG. 9. (a) Divergent and (b) rotational winds at $12.6-\mathrm{km}$ height at $64 \mathrm{~h}, 15 \mathrm{~min}$ into the Gaussian-7.5 simulation. Black $\times$ is the lowlevel center.

Increased divergent kinetic energy alone is not enough to describe the interaction between the TC's outflow and the environment because the outflow has to divert the environmental flow. Upshear outflow causes destructive interference with the environment, while downshear outflow results in constructive interference with the environment to create an outflow jet. Figure 10b shows the difference between upshear and downshear divergent kinetic energy for the G7.5 TC and further 
(a) Volume-Integrated Divergent Kinetic Energy $\left(10^{15} \mathrm{~J}\right)$
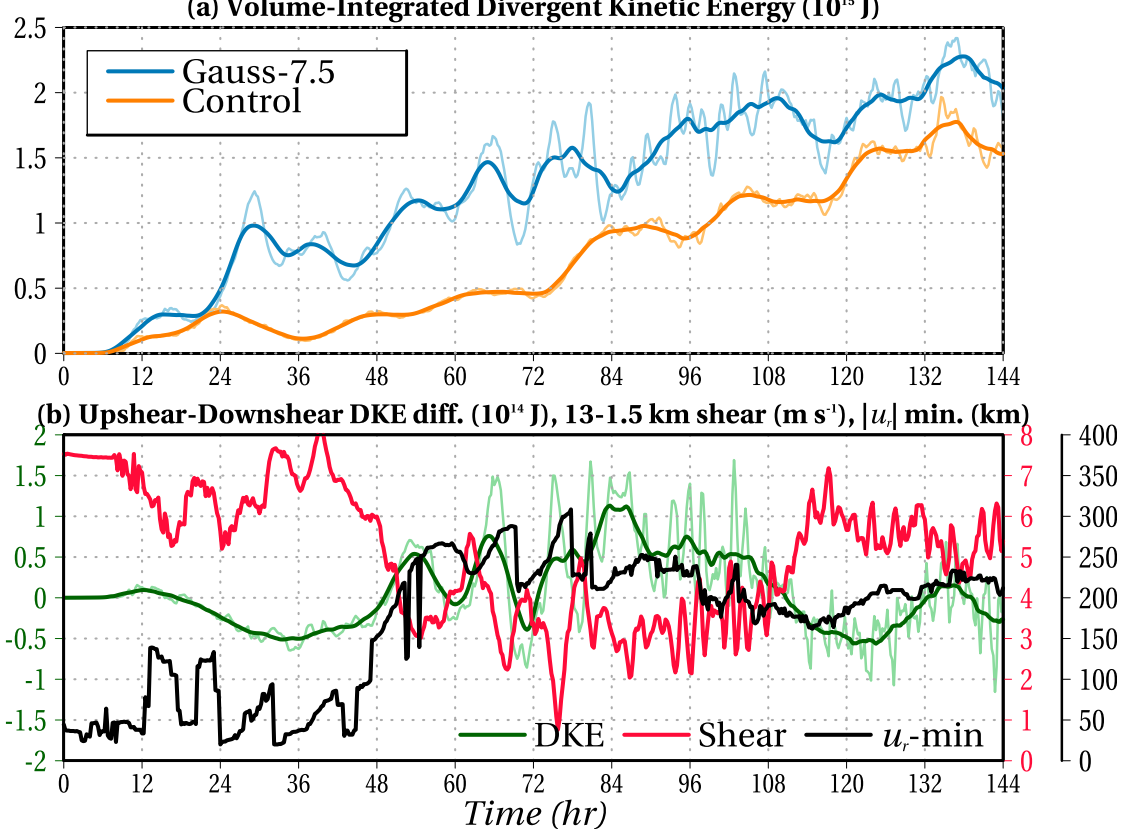

FIG. 10. (a) Volume-integrated divergent kinetic energy (DKE) of the outflow of the sheared (blue) and control (orange) simulations; (b) upshear-downshear differences of DKE (green; where positive, upshear is greater), $13-1.5-\mathrm{km}$ vertical wind shear using a $200-\mathrm{km}$ circle (red), and radial wind magnitude minimum averaged between 13.4 and $12.6 \mathrm{~km}$ (black). For the three DKE time series (blue, orange, green), thin lines are raw values; thick, dark lines are 6-h running means. The shear and the wind minimum time series (red and black, respectively) are unfiltered. The vertical integrations of DKE are between 9 and $16 \mathrm{~km}$.

illustrates the relationship between the outflow front (characterized as the radial wind minimum upshear), the near-core shear, and the upshear-downshear divergent kinetic energy difference. Figure $10 \mathrm{~b}$ demonstrates that, beginning at approximately $48 \mathrm{~h}$, as the precession reaches left-of-shear (see Part II), the energy difference skews upshear, and the shear reduces. Performing a correlation of the shear with the energy difference yields a correlation coefficient of -0.66 . This is a fairly strong inverse correlation, which is higher than the correlation between the total energy and the shear $(-0.52)$ and between the difference and the total energy (0.20). As the divergent kinetic energy difference then skews downshear again after $108 \mathrm{~h}$, the local shear then increases and the intensification also ceases (see Fig. 1b).

This local reduction in shear partly explains why the G7.5 TC intensified; however, the overall sensitivity of the TC to the vertical profile of the environmental winds needs further diagnosis. To address this particular aspect, a trajectory analysis was performed. A series of trajectories were initialized in a $600-\mathrm{km}$ wide line due east of the TC from 10 - to $14.5-\mathrm{km}$ height with $0.5-\mathrm{km}$ vertical spacing and 5-km horizontal spacing, resulting in 120 trajectories per level, at $48 \mathrm{~h}$ into the simulation. The temporal output of the trajectories is $5 \mathrm{~min}$.
Figure 11 shows the trajectories of the parcels initialized at four different levels between 48 and $96 \mathrm{~h}$ of the G7.5 simulation. At 10.5-km height (Fig. 11a), the trajectories are entrained upshear-left of the center. Some of the parcels are forced downward and encircle the eyewall. Most of those parcels that are entrained are ejected into the outflow. At 12.0-km height (Fig. 11b), while there are still indications of entrainment and ejection, there is also evidence of deflection on the southern side of the storm, as evidenced by the trajectories that remain between 10.5 - and 12-km height and enter the outflow jet. A notable shift takes place at $13.0 \mathrm{~km}$ (Fig. 11c), as only four of the 120 trajectories are entrained. At the highest level shown here, $14 \mathrm{~km}$ (Fig. 11d), all of the trajectories indicate deflection. While previous studies (Elsberry and Jeffries 1996; Finocchio et al. 2016) indicate that TCs are sensitive to the depth of the environmental flow, we build on these studies and demonstrate the key aspects of the sensitivity related directly to the outflow interaction with the environmental winds. Figure 11 shows that blocking only occurs in the narrow $(\sim 2 \mathrm{~km})$ 150-250-hPa layer.

When viewed in storm-relative radius-height cross sections, Fig. 12 further shows how the moisture and wind 


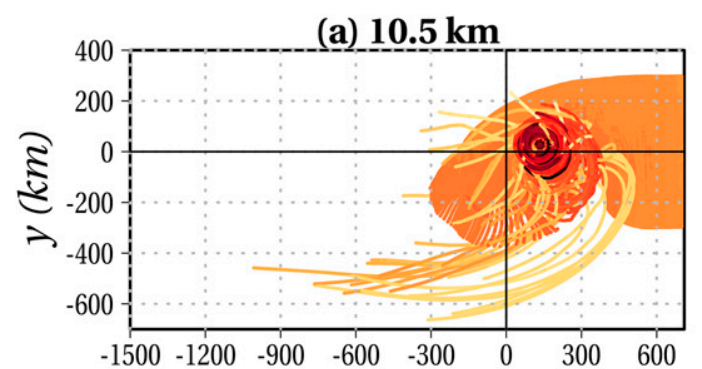

(b) $12.0 \mathrm{~km}$

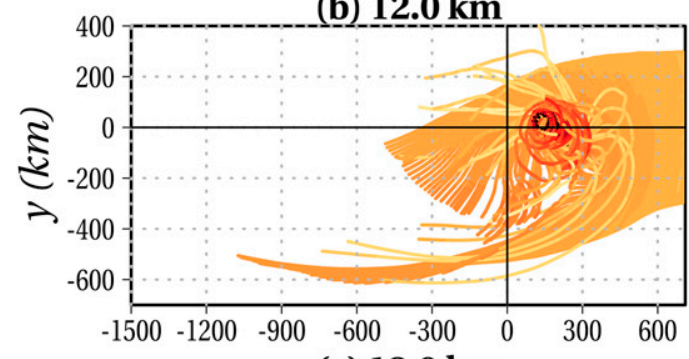

(c) $13.0 \mathrm{~km}$
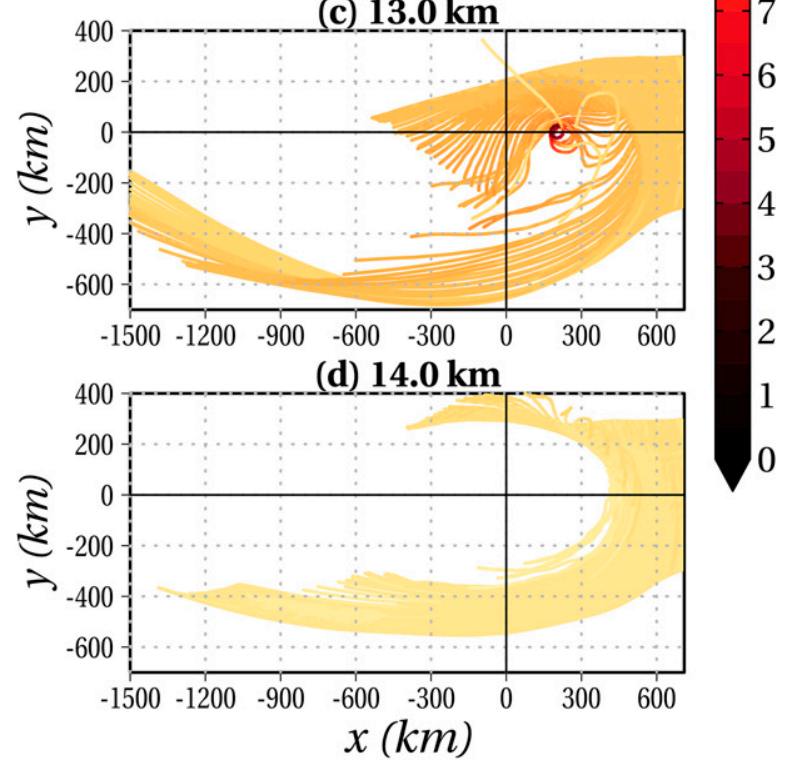

FIG. 11. Evolution of the trajectories with time from 48 to $96 \mathrm{~h}$ of the simulation, launched at (a) $10.5 \mathrm{~km}$, (b) $12.0 \mathrm{~km}$, (c) $13.0 \mathrm{~km}$, and $14.0(\mathrm{~km})$. Trajectories are shaded by height $(\mathrm{km})$ in time.

fields differ around the TC among upshear-right and downshear-left quadrants for both 7.5 TCs and the control. Figure 12a indicates that, downshear-left, outward motion dominates everywhere. In the upshearright quadrant of the G7.5 TC (Fig. 12b), outflow is confined between 12 and $14.5 \mathrm{~km}$. For reference, Fig. 12e illustrates how the outflow is structured in the control simulation. In the C7.5 TC (Figs. 12c,d), there is no radial outflow upshear, and the convection (indicated by higher values of specific humidity change), is $140 \mathrm{~km}$ downshear at this time $(48 \mathrm{~h})$. It is ultimately advected away completely.
Of note here is the localized outflow at $13 \mathrm{~km}$ in the upshear-right quadrant of the G7.5 TC (Fig. 12b) very close to the core in the sheared TC. In terms of vertical velocities and moisture, there is descent at midlevels on the upshear side (Fig. 12b). On both sides of the storm (Figs. 12a,b), and in the control (Fig. 12e), there is drying located beneath the outflow associated with descent. The important distinction among the three figures can be found on the upshear side of the G7.5 TC (Fig. 12b). The descent region begins higher in the environment- $13.5 \mathrm{~km}$ upshear compared with $11.5 \mathrm{~km}$ downshear and in the controland is also of larger magnitude. Of great importance is the moisture profile. Figure $12 \mathrm{~b}$ shows that the moistening is only occurring at $14-\mathrm{km}$ height (i.e., the tropopause) beyond the outflow and drying virtually everywhere else. This tropopause moistening radially beyond the outflow is very small $(\sim 10 \%)$. The drying also occurs much closer to the storm on the upshear side in the G7.5 TC than in the control, and the magnitude of the drying is twice as large as the downshear location. This appears to be associated with environmental sinking and not TC outflow, since according to the trajectories, when the parcels are ejected into the outflow, they remain at a high altitude in the troposphere.

\section{c. Remote upshear effects}

We now examine Regions II and III from Fig. 2, which is also the region of the WV clearing discussed in Part I. Figure 13 shows divergent and rotational winds averaged over $2.5 \mathrm{~h}$, when the radial wind zero line is near its greatest extent (Fig. 4c), of winds and pressure. These fields are also averaged over a $20-\mathrm{km}$ meridional band due east of the vortex center; therefore, zonal winds are de facto radial winds. The total zonal wind changes direction at approximately $280 \mathrm{~km}$ from the low-level vortex center. This figure also clearly shows that the TC affects the environmental flow $1000 \mathrm{~km}$ upstream: this is the bow wave.

Deceleration beyond the outflow front is due to a small outward pressure gradient force. A dynamic high pressure exists near the outflow front. The existence of this higher pressure region can be explained using Bernoulli's equation:

$$
p_{t}=p_{s}+p_{d}
$$

where $p$ is pressure and the subscripts abbreviate total $(t)$, static $(s)$, and dynamic $(d)$. Static pressure is the hydrostatic background. The dynamic pressure is simply the change in kinetic energy of the flow: $(1 / 2) \rho(\Delta v)^{2}$. Using environmental values at $13-\mathrm{km}$ height in (3) 
(a) Gaussian-7.5, Downshear Left

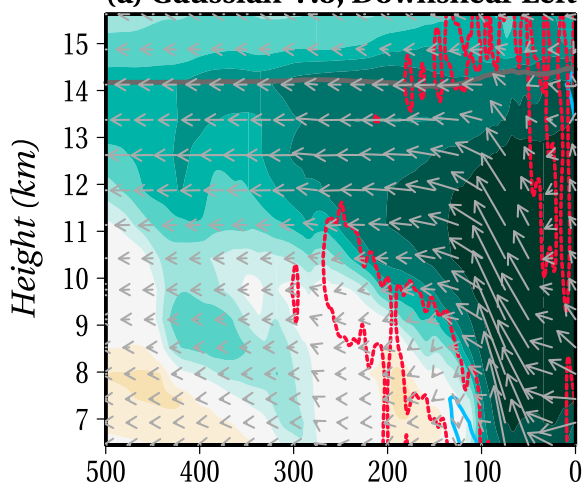

(c) Cosine-7.5, Downshear Left

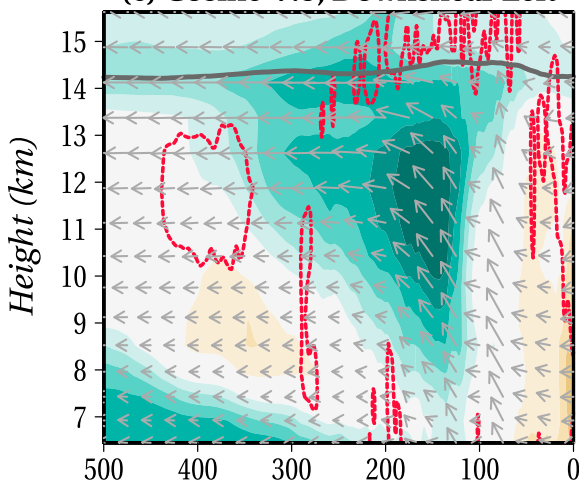

(e) Control, Symmetric

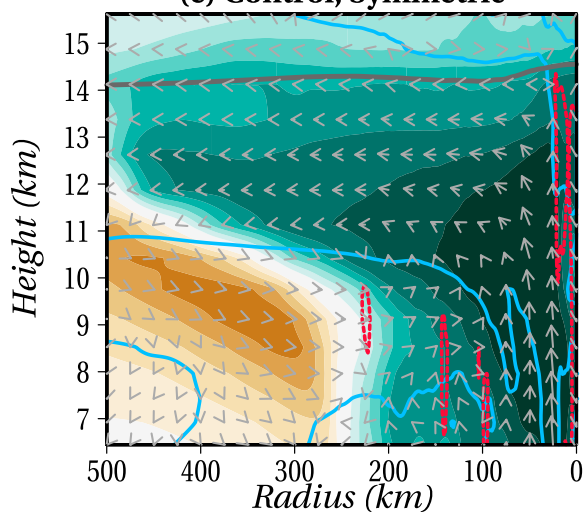

(b) Gaussian-7.5, Upshear Right

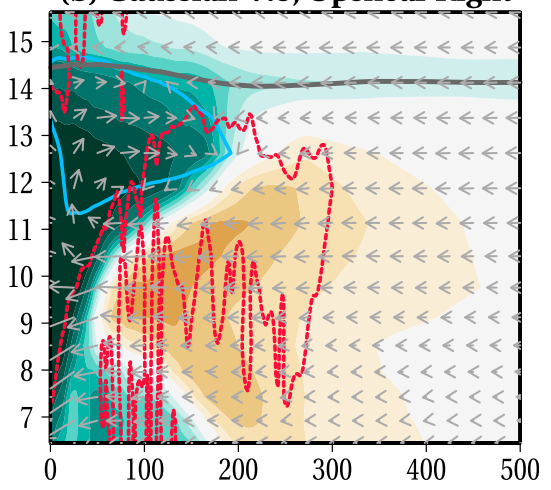

(d) Cosine-7.5, Upshear Right
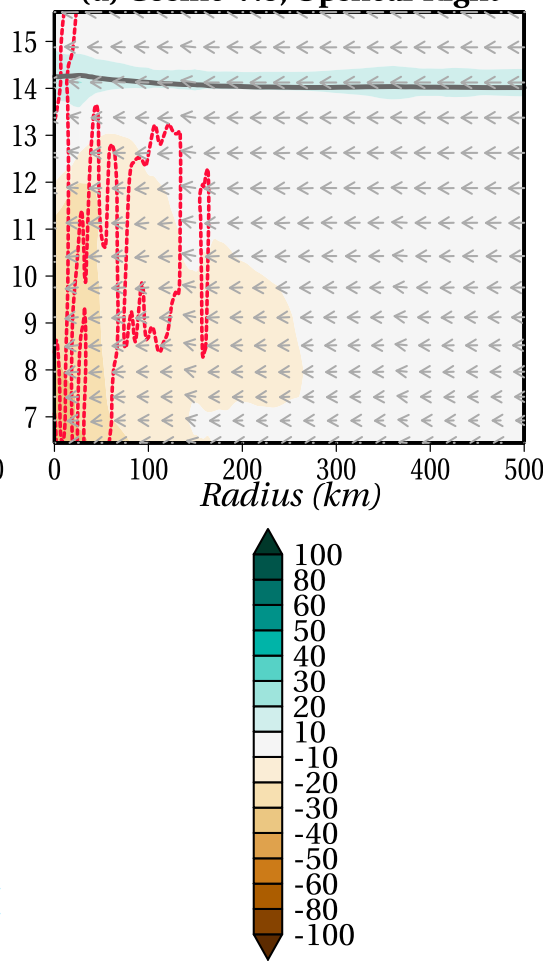

FIG. 12. Percent change in specific humidity from initialization time (\%, shaded), tropopause $\left(N^{2}=2 \times 10^{-4} \mathrm{~s}^{-1}\right.$, thick gray line), radial wind zero line (blue contour), vertical velocity $-0.02 \mathrm{~m} \mathrm{~s}^{-1}$ contour (dashed red), and radial-vertical wind vectors for the (a) downshear-left and (b) upshear-right quadrants for the Gaussian-7.5 TC, for the Cosine7.5 TC's (c) downshear-left and (d) upshear-right quadrants, and (e) for the symmetric fields for the control. For the vectors, vertical winds are multiplied by 20 . All are averaged with a 2.5-h running mean. Gaussian-7.5 and Control images are taken from when the two TCs are of equivalent low-level strength: $63 \mathrm{~h} 00 \mathrm{~min}$ for the sheared TC, $72 \mathrm{~h} 00 \mathrm{~min}$ for the control TC. Cosine-7.5 images occur at $48 \mathrm{~h}$.

results in a dynamic pressure perturbation of approximately $10 \mathrm{~Pa}$ from the environment. To determine the dynamic pressure on the outflow side, we use a value of $6 \mathrm{~m} \mathrm{~s}^{-1}$, since that is the value at which the divergent winds and the total winds diverge $150 \mathrm{~km}$ away from the center. This results in a pressure increase of $5 \mathrm{~Pa}$, for a total increase of $15 \mathrm{~Pa}$. The total increase in pressure from the environment to the peak at the outflow front is approximately $17 \mathrm{~Pa}$. We realize that the winds change direction rather than strictly reduce to zero, and there is an inherent incompressibility assumption in (3) (Darrigol and Frisch 2008); nonetheless, 


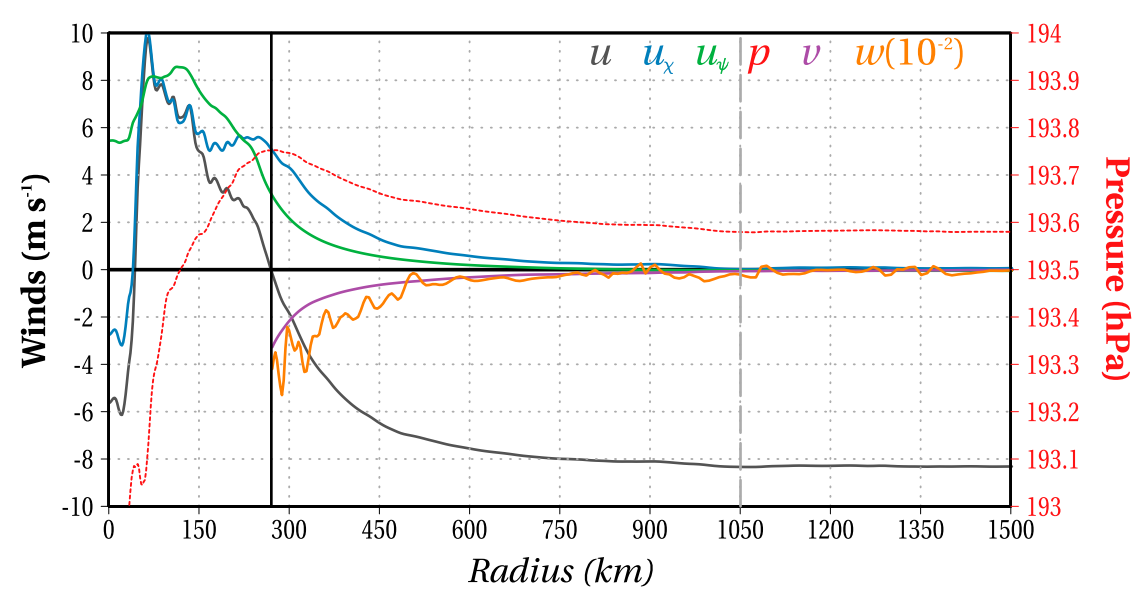

FIG. 13. Upshear total zonal winds, divergent zonal winds, rotational zonal winds, vertical velocity (scaled by a factor of 100), total meridional winds (all left axis), and pressure (right axis) at $12.6 \mathrm{~km}$ at $68 \mathrm{~h}$ into the model simulation. Fields are time averaged over $2.5 \mathrm{~h}$ and are meridionally averaged over $20 \mathrm{~km}$ due east of the low-level TC center. Distance is from lowlevel center location. Vertical black line indicates the location of the outflow front (where total zonal winds change direction). Vertical dashed gray line is the bow wave (where environmental winds begin decelerating).

this simple analysis accounts for the pressure increase. ${ }^{2}$ The bow wave can then be described as the farthest outward extent of the pressure gradient force caused by this high pressure.

To separate this feature from the larger anticyclone typically associated with a TC and then advected away in shear (Wu and Emanuel 1993, 1994), we refer to this upshear local increase as a "Bernoulli high" whose magnitude is not static in time. Figure 13 illustrates the high at $68 \mathrm{~h}$ when the outflow front is at a local maximum. Figure 14 is a planar view of the pressure field $1 \mathrm{~h} 15 \mathrm{~min}$ earlier in order to demonstrate three things: the dual-lobe nature of the higher pressures aloft, the Bernoulli high's magnitude change in time, and the Bernoulli high's location. When the outflow front is expanding (the divergent kinetic energy is increasing), the high strengthens and moves inside the front, which we have simply approximated with the zero-radial-wind line. While the environmental portion of the pressure increase remains fairly constant, the outflow portion will change, and that changes the structure of the pressure field as the kinetic energy dissipates.

As noted in Fig. 13, the environmental winds slow down as they approach the outflow front. This results in a large area of convergence upwind of the front. Owing to the fact that this interface is buttressed against

\footnotetext{
${ }^{2}$ This phenomenon is a divergent-outflow-specific manifestation of the Magnus effect, which itself is a special case of Bernoulli's principle. We investigate this phenomenon further in a future manuscript.
}

the tropopause, this convergence results in subtle sinking that increases in magnitude the closer it gets to the outflow front (Figs. 12b and 13). Based on the trajectory analysis in section $3 b$, it has been shown that parcels in the outflow maintain their heights and do not sink upwind, further confirming our assertion that it is the environment - and not the outflow-sinking on the upshear side of the TC.

Since the environment is initialized in geostrophic thermal wind balance, ageostrophic theory dictates that when the zonal geostrophic wind decelerates, it is deflected to the left; hence, the meridional wind becomes negative (Holton 1992, 175-176). Mathematically, this can be expressed by the meridional momentum equation, neglecting subgrid-scale terms:

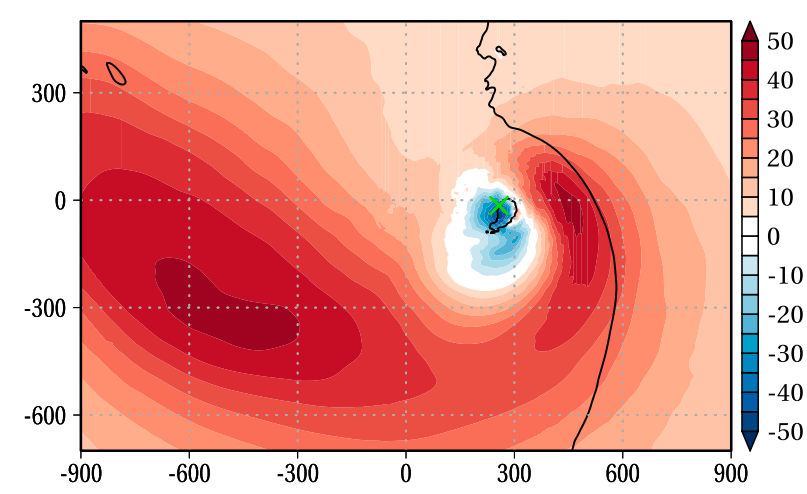

FIG. 14. Planar view of pressure perturbation (Pa) at $66 \mathrm{~h} 45 \mathrm{~min}$ at $12.6 \mathrm{~km}$. Contour is zero radial wind line. All fields are smoothed with a two-hour running mean. " $X$ " is low-level TC center. Axes are $\mathrm{km}$ from domain center. 


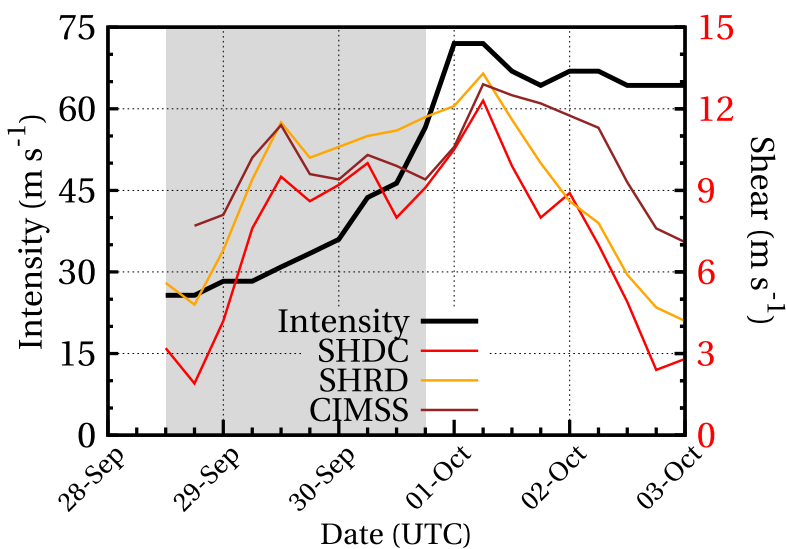

FIG. 15. Intensity (left axis) and shear (right axis) time series of 2016 NATL Matthew. SHDC and SHRD are from the SHIPS database. Gray box indicates time from genesis to appearance of eye in IR imagery.

$$
\frac{D v}{D t}+f u=-\frac{1}{\rho} \frac{\partial p}{\partial y} .
$$

The prescribed easterly shear creates a high pressure on the northern side of the domain (not shown). Since the Coriolis acceleration of the now-decelerated zonal environmental winds no longer balances the pressure gradient imposed by the thermal wind balance, there will be a southward acceleration quantified as $-f u^{\prime}$, where $u^{\prime}$ is the positive and eastward deviation from the negative and westward background as far as the pressure gradient extends into the flow. A possible explanation for the radial scale of the pressure gradient can be determined by using the definition of the baroclinic Rossby deformation radius:

$$
L_{R}=\frac{N H}{n \pi f}
$$

The radius of the $n=1$ mode, given the environmental parameters of our simulation $\left(N=0.01 \mathrm{~s}^{-1}, H=14 \mathrm{~km}\right.$, $\left.f=0.00005 \mathrm{~s}^{-1}\right)$, is $891 \mathrm{~km}$. This is approximately the distance at which the environmental wind decelerates and deviates from geostrophic balance upwind of the Bernoulli high. While not absolutely definitive, this calculation does indicate a potential reason for why the environmental winds begin slowing $1000 \mathrm{~km}$ upwind of the TC.

\section{Verification and operational applications}

\section{a. Part I "verification"}

In Part I, two features of the atypical RI class of TCs identified in WV imagery were upshear arcs and upshear clearing/warming. The most significant finding in this manuscript of the upper-level behavior is that the outflow serves to divert the environmental winds, thus reducing the local shear felt by the core of the vortex. The arcs, which are slow-moving wavelike features, serve as visual approximations of this flow diversion. We have shown that the shear actually was reduced by the TCs themselves, but the shear calculation methods used operationally, which use much larger areas for shear and divergence calculations, were probably not capturing this effect. Our analysis has identified weaknesses in such approaches, as the block is directly related to the divergent wind and occurs within $300 \mathrm{~km}$ radially. Furthermore, outflow far upshear is not required, as upshear outflow only needs to divert the upshear environmental winds sufficiently to allow for vortex realignment. Historically, outflow for TCs undergoing RI is generally thought to organize itself into two different outflow jets (Merrill and Velden 1996), but atypical RI TCs only require one.

Part I documented a nearly stationary wavenumber-1 Fourier anomaly in the far-field WV brightness temperatures in Norbert and Joaquin (Fig. 18 from Part I). We can now explain why this anomaly persists. The near-permanent wavenumber-1 asymmetry observed in far-field WV imagery is a manifestation of the slowed and sinking environmental wind at upper levels on the upshear side described herein associated with convergence due to the Bernoulli high.

\section{b. Additional operational application}

All of the features presented in section 3 are physically relevant to explain atypical RI and the associated evolution on satellite, but some features (the blocking and the arcs) are easier to observe than others (the bow wave). Since this series was motivated by satellite observations, it is worthwhile to demonstrate how this work would apply to an operational forecast. For demonstration, we choose a high-impact atypical RI TC that was not part of the original six from Part I but nevertheless underwent RI in moderate vertical wind shear: 2016 northern Atlantic (NATL) Matthew (Stewart 2017). For this analysis, we use SHIPS and CIMSS shear analyses, GOES-13 WV observations, CIMSS AMVs, and $0.5^{\circ}$ GFS analyses. We use the GFS analyses here in an attempt to simulate operational conditions more closely. The decomposition of the GFS analyses into rotational and divergent components is performed using SPHEREPACK (Adams and Swarztrauber 1997).

Matthew intensified in shear ranging between 9 and $11 \mathrm{~m} \mathrm{~s}^{-1}$ according to operational shear calculations (Fig. 15). Of note here is that the shear possesses a westerly component, which we will illustrate shortly, a fact that is unique when compared with the easterly 
(a) $2016-09-300145$ UTC

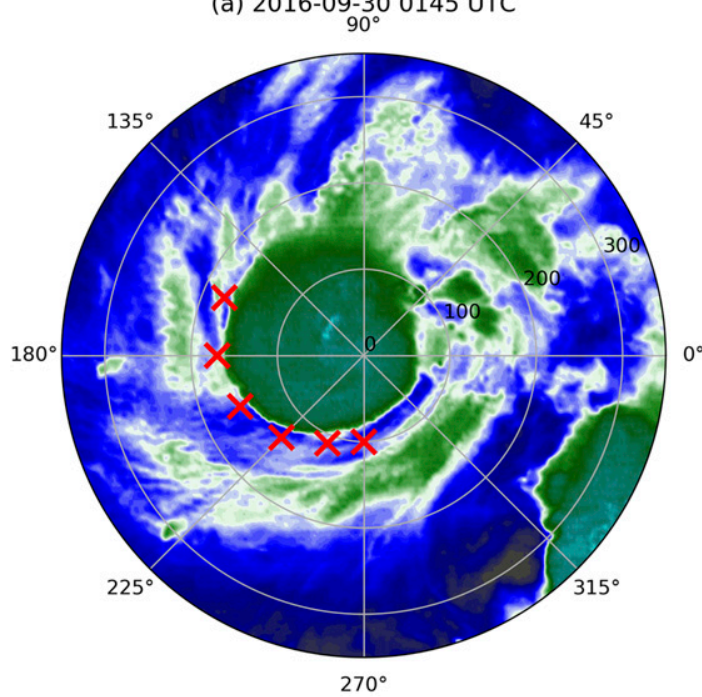

(c) 2016-09-30 0315 UTC

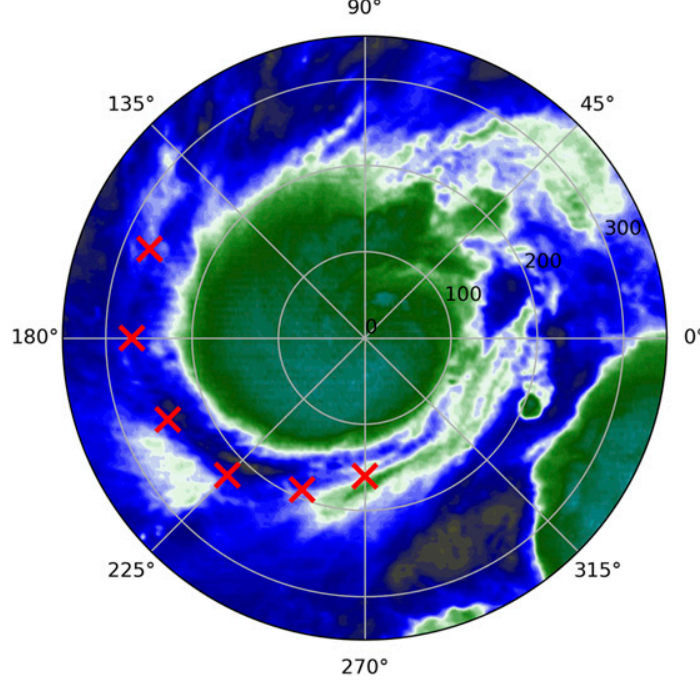

(b) $2016-09-300245$ UTC

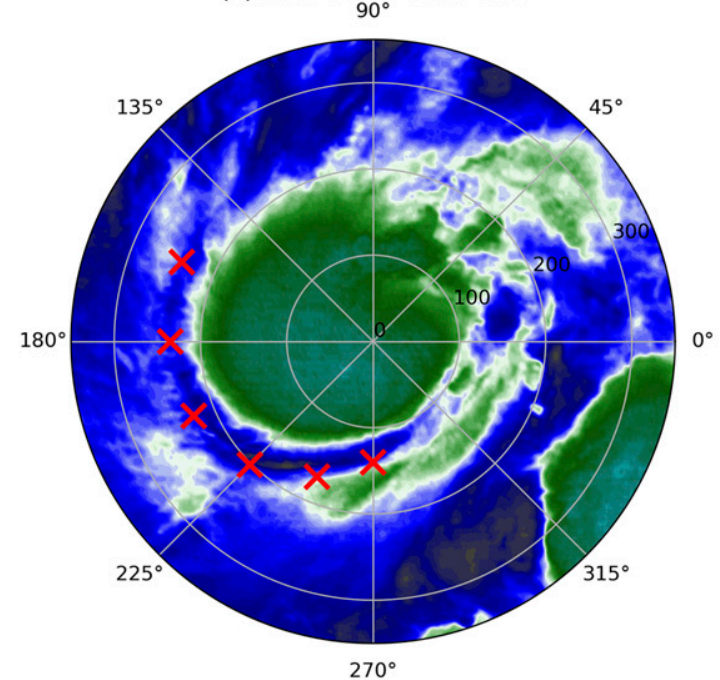

(d) 2016-09-30 0345 UTC

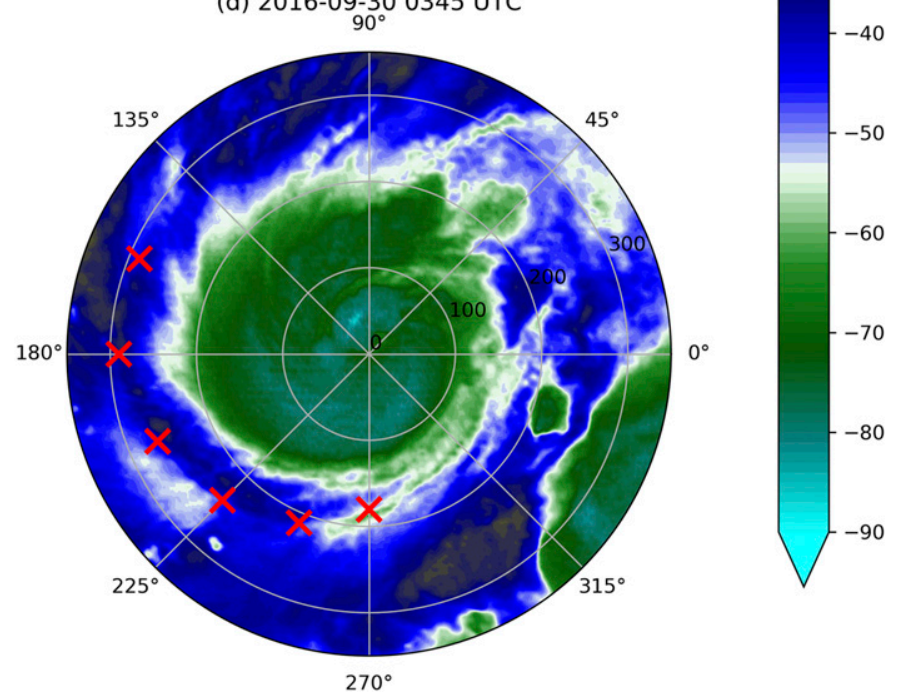

FIG. 16. Storm-relative WV satellite imagery of 2016 NATL Matthew at (a) 0145, (b) 0245, (c) 0315, and (d) 0345 UTC 30 Sep. Red $\times$ s mark the outward propagating arc/gap. Angles are math convention $\left(0^{\circ}\right.$ is east, $90^{\circ}$ is north, etc.); radial distances are in $\mathrm{km}$.

shear of the northeastern Pacific (EPAC) TCs, and even NATL Joaquin, from Part I. Operationally, Brown (2016) noted on 29 September: "Given the current shear and structure of Matthew, only slight strengthening is predicted during the next $24 \mathrm{~h}$." Approximately $24 \mathrm{~h}$ later on 30 September, after an intensity increase of $20 \mathrm{~m} \mathrm{~s}^{-1}$, Brennan (2016) noted, "I am reluctant to show a decrease in intensity given that the environment around the cyclone does not appear to change much." In the postseason Tropical Cyclone Report, Stewart (2017) wrote: "The aforementioned remarkable RI period, which has only been exceeded a few times in the Atlantic historical record, occurred during apparently unfavorable environmental conditions consisting of west-southwesterly 850-200-mb vertical wind shear of
18-22 kt." The high shear appears to be the source of uncertainty in physically explaining Matthew's behavior.

For our discussion, we bypass the IR/tilt analyses of Parts I and II in order to focus on the outflow blocking described within this manuscript. Figure 16 is a series of successive WV satellite images of a TCA early on 30 September and the warm gap plus the arc on the western (upshear) side of the TC (see section 3b). By 0315 and 0345 UTC (Figs. 16c,d), the gap/arc, marked by the series of red $\times \mathrm{s}$, indicates that the outflow has pushed back at least $250 \mathrm{~km}$ from the center of Matthew to the west-southwest. Conclusive AMV proof of how far the outflow reaches can be seen at 1800 UTC 30 September. Figure 17a illustrates the wind barbs and the zero radial wind line at this time. Southwest of the 
(a) 2016-09-30 1800 UTC

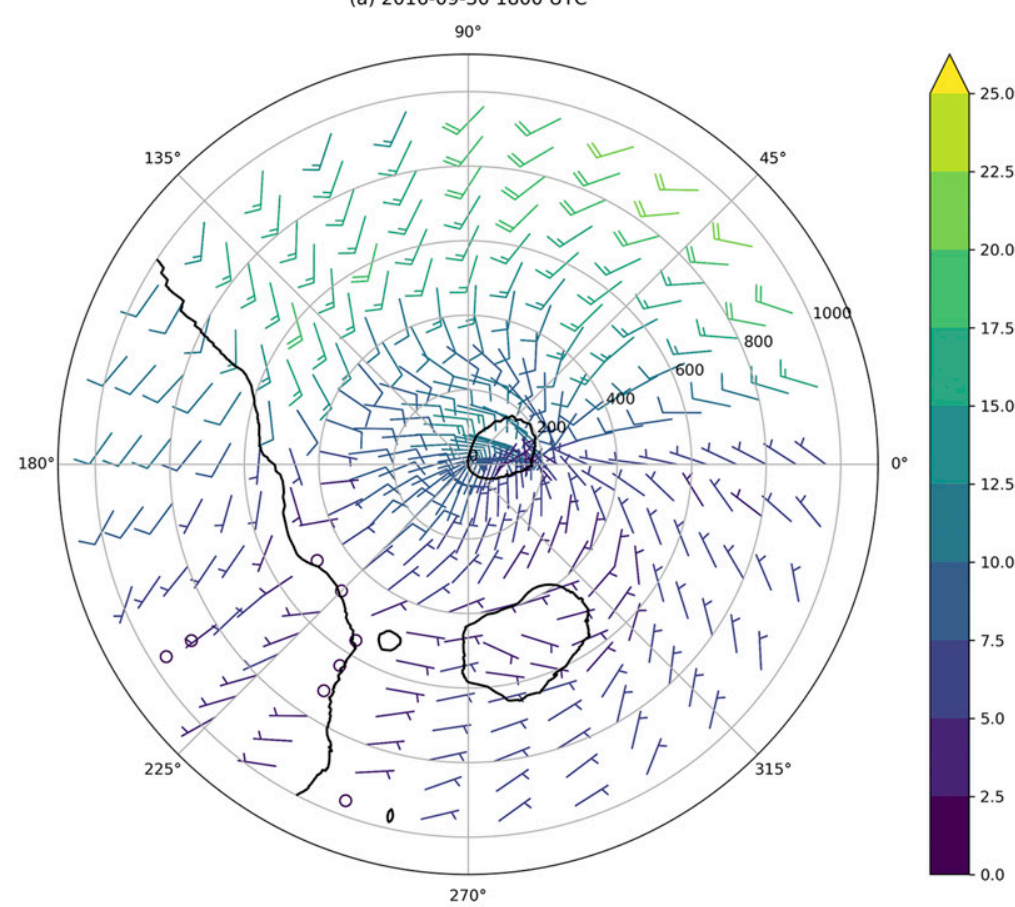

(b) 2016-09-30 0600 UTC

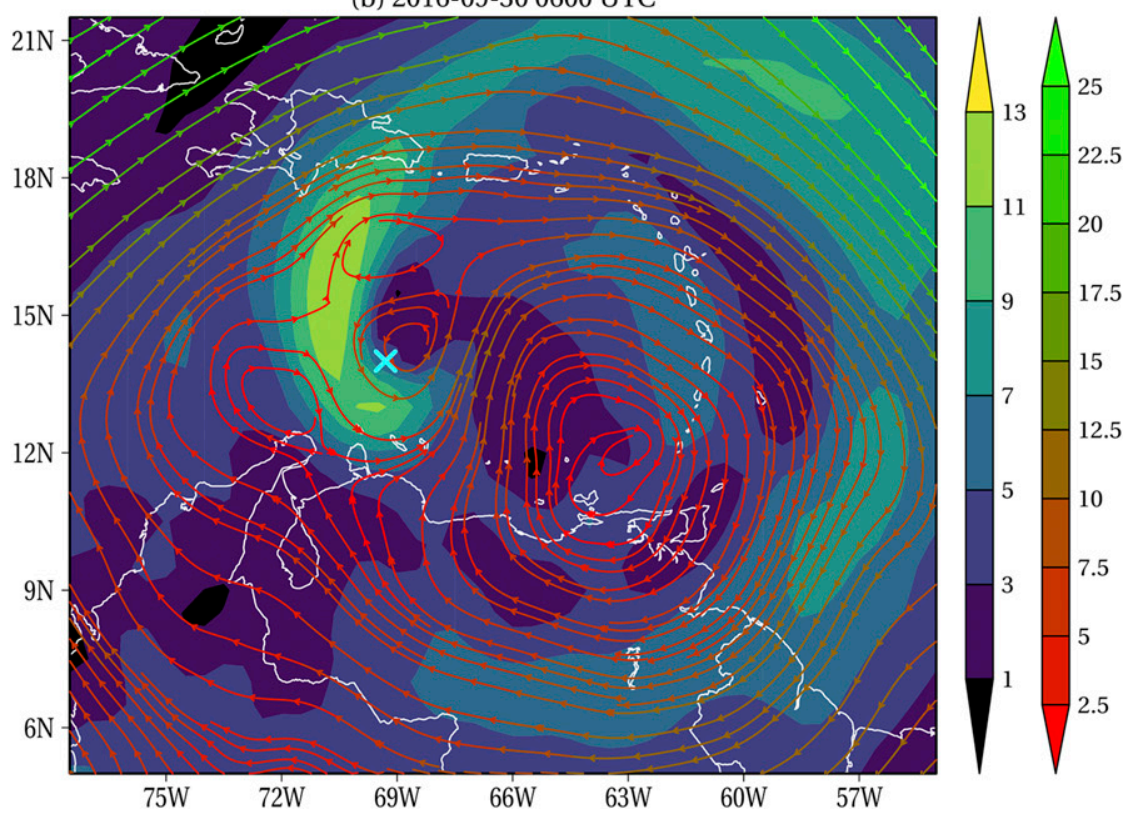

FIG. 17. (a) Interpolated AMVs from $250 \mathrm{hPa}$ and higher around Matthew's center at 1800 UTC 30 Sep. Black line is radial wind zero line. (b) Divergent wind magnitude (shading) and rotational winds (streamlines) at $200 \mathrm{hPa}$ near Matthew (cyan $\times$ ) derived from the GFS analyses at 0600 UTC 30 Sep. All units are $\mathrm{m} \mathrm{s}^{-1}$.

TC, the outflow has pushed the oncoming environmental winds back approximately $500 \mathrm{~km} .12 \mathrm{~h}$ earlier, using a more sophisticated data assimilation system, the GFS demonstrates a rotational-divergent wind structure at $200 \mathrm{hPa}$ that is similar to the analyses presented in section 3b. Specifically, Fig. 17b illustrates a divergent wind maximum on the upshear (western) portion of the TC, where the cyan $\times$ marks the best track position. The rotational winds indicate that Matthew was under the influence of a large upper-level anticyclone, 
fulfilling a necessary environmental condition of Part I, whose center was to Matthew's southeast. Most importantly, the rotational winds also indicate that Matthew's divergent outflow is rerouting the environmental winds around the storm, as the bifurcation of the environmental flow exists where the divergent winds from the outflow are at their near-core local maximum. This flow diversion occurs approximately $150-200 \mathrm{~km}$ away from the TC center and suggests that the shear calculations all missed this effect (Fig. 15). Given this analysis, we assert that Matthew underwent atypical RI, and the earliest indication that it is conclusively happening is 0600 UTC 30 September when Matthew was a borderline hurricane. While we realize this is and was a challenging forecast, we recommend that forecasters use the analytical tools available to make these difficult decisions when nearly all of the model guidance is incorrect.

\section{Summary and discussion}

\section{a. Summary}

The upper levels of a rapidly intensifying tropical cyclone (TC) in hostile environmental wind shear conditions have been analyzed using a compressible, nonhydrostatic model (CM1). Upper-level wind analyses indicate that the outflow from convection, quantified as the kinetic energy of the divergent wind, serves to divert and to block the environmental flow at upper levels. This happens in two phases. The first part requires a small region (on the order of $50 \mathrm{~km}$ upshear) be diverted upshear until the TC tilt reaches left-of-shear. Following that, the nutations become the dominant modulator of convection, and the cumulative effect of the repeating convective events is to push the upshear environmental winds back out to a radius of $200 \mathrm{~km}$ from the TC center, thus reducing the local shear felt by the vortex. These effects allow for this TC to realign itself. After processing the CM1 output through the Community Radiative Transfer Model (CRTM), we demonstrated that the CM1 produces thin cloud arcs that are separate from the main cloud shield on the upshear side, phenomena that are also present in satellite observations. These arcs exist between 12 and $14 \mathrm{~km}$, and they serve as visual guidance for the extent of the blocking. They are caused by slow-moving wavelike features with a radial wavelength of $50 \mathrm{~km}$ and a speed of $8 \mathrm{~m} \mathrm{~s}^{-1}$.

The upper levels upwind of the TC center can be divided into three regions: the outflow, the environmental deceleration region, and the free atmosphere. The outflow front is where the outflow and the environment meet, and it can be defined by where the radial wind changes direction and by a sharp drop in relative humidity. Beyond the front lies a large area (250$1000 \mathrm{~km}$ radially outward) of convergence where the environmental flow is decelerated by a dynamic (Bernoulli) high created by the block. Physically, the wind here curves to the left and slowly sinks. This is the source of the far-field WV temperature anomaly identified in Part I. Far beyond the outflow front $(\sim 1000 \mathrm{~km})$ is the bow wave, which is the extent of the dynamic high pressure upshear, beyond which are unaffected environmental winds.

Finally, the trajectory analysis indicates why the depth of the flow is so critical to modulating TC intensification: the outflow can only block winds in a narrow layer below the tropopause that is $100 \mathrm{hPa}$, or $2 \mathrm{~km}$, deep. Elsberry and Jeffries (1996) hypothesized that due to the diurnal forcing, the outflow of Typhoon Omar acted to divert the environmental winds around it. Here, we have related the flow diversion to outflow from convection associated with the tilt of the TC, but this effect is only valid under certain conditions: specifically, when the shear is due to vertically shallow upper-level anticyclones. Conversely, winds associated with troughs extend more deeply into the atmosphere, and the outflow blocking mechanism described herein can do nothing about environmental winds between 300 and $500 \mathrm{hPa}$, for example. Operationally, we believe that Velden and Sears (2014) and Knaff et al. (2018) performed important steps in attempting to account for this issue by using layer averages instead of singular levels and by vertically integrating environmental winds, respectively, but a deeper analysis of the issue may still be necessary.

\section{b. Synthesis, discussion, and future work}

Over the course of this set of three articles, we have endeavored to describe the phenomenology and morphology of a class of TCs that undergo a moderateshear-induced, "atypical" pathway to RI, focusing on the broader key physical concepts and operational utility in lieu of mathematical rigor. Generally speaking, using the parlance of Kaplan et al. (2010), Rozoff et al. (2015), and Knaff et al. (2018), this behavior occurs when the atmosphere is otherwise favorable thermodynamically, but the perceived limiting factors are the shear and the upper-level divergence. Operationally, a forecaster would have to ask him/herself three basic questions: 1) Are the lower levels (including SSTs) warm and moist? 2) What is the shear value? 3) Is the TC being sheared by an anticyclone? Obviously, we still must perform a full climatological analysis to identify TCs that exhibit TCAs and characteristics of blocking but do not develop or develop slowly. Nevertheless, these three simple questions, plus the satellite markers discussed throughout this series indicating tilt nutations 
and outflow blocking, should act as a convenient alert for forecasters to at least be aware that the guidance may be problematic. The ability to perform a rotationaldivergent decomposition on the wind field would further indicate the hallmarks of this behavior, although we would point out that in a real-world model, multiple divergent sources could potentially exist, and a DKE integration over a storm-relative domain would be advisable.

We make the important point here that the shear reduction discussed throughout the manuscript is different from the shear reduction discussed in Zhang and Tao (2013) and in Rappin and Nolan (2012). Zhang and Tao (2013) argue that the vertical wind shear increase and then subsequent decrease between 900 and $500 \mathrm{hPa}$ is related to intensification. In their calculations, shear increases from a background value of 2 to $6 \mathrm{~m} \mathrm{~s}^{-1}$ before reducing again to $2 \mathrm{~m} \mathrm{~s}^{-1}$. In our simulations, this value is practically zero in both Gaussian-shear initial conditions (further discussed in the appendix). Zhang and Tao (2013) state that the increase in shear is due to environmental forcings, but they never discuss why the shear increases and subsequently decreases. We have shown that for our TCs, it is definitively the outflow that is causing near-core shear modulation at upper levels. Our findings echo examples of blocking from convective outflow described in other studies (Fritsch and Maddox 1981; Schmidt and Cotton 1990; Trier and Sharman 2009), but those studies focused on midlatitude squall lines and mesoscale convective systems. We believe we have isolated the same basic effect, only on a scale 10-20 times larger.

We believe our analyses raise a host of questions about atypical RI and perhaps TCs in general, which we hope to address in future work, including but not limited to the following:

- How often does this behavior happen worldwide? As NATL Matthew was brought to our attention externally (M. DeMaria 2019, personal communication), so were two Australian cyclones: 2017 Ernie and 2019 Riley (J. Courtney 2019, personal communication). All three were in moderate-to-high shear caused by upper-level anticyclones and unexpectedly intensified (RI occurred for both Matthew and Ernie). All exhibited features documented in this series. What are the environmental bounds (i.e., SSTs, shear magnitudes) that govern when this process occurs, when this process fails, and when a TC intensifies by more "classical" methods?

- Why is the divergent outflow two to three times greater in the G7.5 TC than in the control? How do the changes in the thermodynamic structure, as noted in Part II, affect this behavior? How would changes in SSTs and the diurnal cycle affect the convection and, subsequently, the outflow?

- Why does this shear profile result in a tilt at all? Why is the blocking effective? See the appendix for more specific details about this question.

Evidently, many questions remain, and these are only but a few questions that we believe our research has raised. Much more work remains to be done.

Acknowledgments. David Ryglicki graciously acknowledges a National Research Council Postdoctoral Research Award and a Karles Fellowship Award at the Naval Research Laboratory in Monterey, California. This research was supported by the Office of Naval Research as part of the Tropical Cyclone Intensity Departmental Research Initiative (PE 0601153N). James Doyle and Jerome Schmidt also acknowledge the support of the Chief of Naval Research through the NRL Base Program (PE 0601153N). David would like to acknowledge George Bryan for making the CM1 freely available. He would also like to acknowledge Russ Elsberry for his time and helpful discussions. Finally, we would like to acknowledge John Knaff, Chris Velden, and one anonymous reviewer, as well as Editors David Schultz and Ron McTaggart-Cowan, for their collective patience and helpful comments that greatly improved the readability, clarity, and content of all three parts.

\section{APPENDIX}

\section{Vortex Tilt Relative to Differential Advection}

A question posed in section $5 \mathrm{~b}$ concerns the tilt of the TC relative to the background environmental winds. Specifically, prevailing theory (Reasor and Montgomery 2015; Reasor et al. 2004; Schecter et al. 2002; Jones 1995) indicates that vortex tilt is due to differential advection in the vertical by the background wind. Induced flow from the tilting layers is then thought to reduce this tilt by self-advection (the precession); however, in the G7.5 case, we document the reverse. Figure A1 shows the smoothed-in-time tilt of the G7.5 vortex and what the tilt should be if the vortex were simply being forced by advection $24 \mathrm{~h}$ into the simulation. From $2-$ to $7.5-\mathrm{km}$ height, the tilt is greater than the advective forcing at this time. While ascertaining the specifics of this result are far beyond the scope of this paper, we show this to demonstrate that even though the outflow blocking arguments described throughout this manuscript only affect a narrow layer, our analysis indicates that the evolution of the tilt is more complex than discrete 


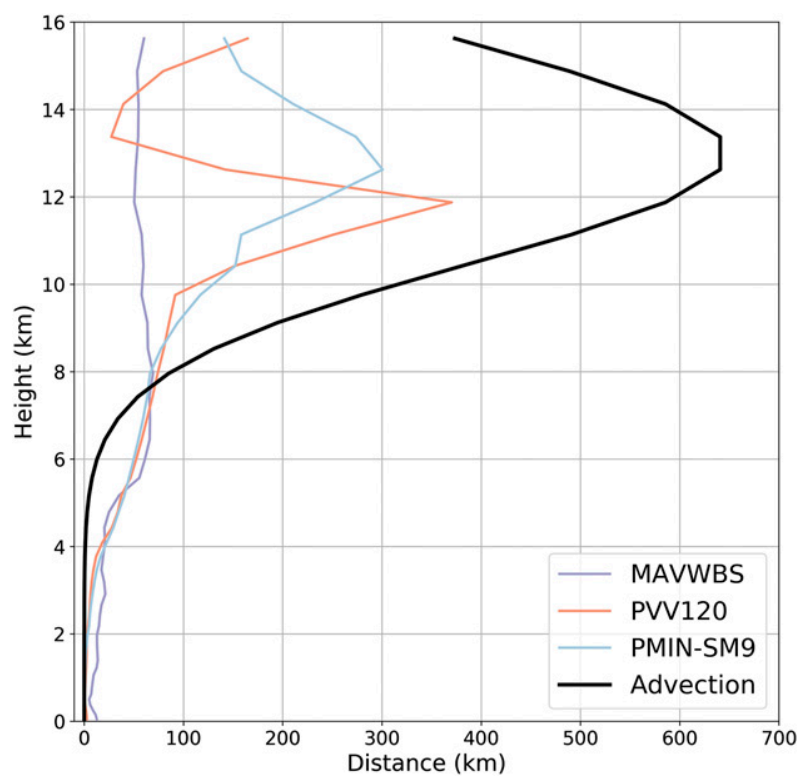

FIG. A1. Tilt magnitudes with height using three different centerfinding methods (see Table 1) and what the tilt would be were it forced solely by differential advection (Advection) at $24 \mathrm{~h}$ into the G7.5 simulation.

level-to-level differential advection arguments. If the upper-level winds are indeed causing the vortex to tilt at lower levels, then the blocking and the reduction of the force of the winds aloft would also theoretically affect the evolution of the tilt several kilometers below.

\section{REFERENCES}

Adams, J., and P. Swarztrauber, 1997: SPHEREPACK 2.0: A model development facility. NCAR Technical Note TN436+STR, 62 pp., https://doi.org/10.5065/D6Z899CF.

Ahern, K., and L. Cowan, 2018: Minimizing common errors when projecting geospatial data onto a vortex-centered space. Geophys. Res. Lett., 45, 12 032-12 039, https://doi.org/10.1029/ 2018 GL079953.

Bikos, D., and Coauthors, 2012: Synthetic satellite imagery for realtime high-resolution model evaluation. Wea. Forecasting, 27, 784-795, https://doi.org/10.1175/WAF-D-11-00130.1.

Black, M. L., J. F. Gamache, F. D. Marks Jr., C. E. Samsury, and H. E. Willoughby, 2002: Eastern Pacific Hurricanes Jimena of 1991 and Olivia of 1994: The effect of vertical shear on structure and intensity. Mon. Wea. Rev., 130, 2291-2312, https://doi.org/10.1175/1520-0493(2002)130<2291:EPHJOA> 2.0.CO;2.

Black, P. G., and R. A. Anthes, 1971: On the asymmetric structure of the tropical cyclone outflow layer. J. Atmos. Sci., 28, 1348-1366, https://doi.org/10.1175/1520-0469(1971)028<1348: OTASOT $>2.0 . \mathrm{CO} ; 2$.

Bluestein, H. B., 1992: Synoptic-Dynamic Meteorology in Midlatitudes. Vol. II: Observations and Theory of Weather Systems. Oxford University Press, 608 pp.

Bosart, L. F., W. E. Bracken, J. Molinari, C. S. Velden, and P. G. Black, 2000: Environmental influences on the rapid intensification of Hurricane Opal (1995) over the Gulf of Mexico. Mon. Wea. Rev., 128, 322-352, https://doi.org/10.1175/ 1520-0493(2000)128<0322:EIOTRI >2.0.CO;2.

Brennan, M., 2016: Hurricane Matthew discussion number 10. NWS TPC/National Hurricane Center, Miami, FL, accessed 20 January 2019, https://www.nhc.noaa.gov/archive/ 2016/al14/al142016.discus.010.shtml.

Brown, D., 2016: Tropical Storm Matthew discussion number 5. NWS TPC/National Hurricane Center, Miami, FL, accessed 20 January 2019, https://www.nhc.noaa.gov/archive/ 2016/al14/al142016.discus.005.shtml.

Bryan, G. H., and J. M. Fritsch, 2002: A benchmark simulation for moist nonhydrostatic numerical models. Mon. Wea. Rev., 130, 2917-2928, https://doi.org/10.1175/1520-0493(2002)130<2917: ABSFMN $>2.0 . \mathrm{CO} ; 2$.

Cressman, G. P., 1959: An operational objective analysis system. Mon. Wea. Rev., 87, 367-374, https://doi.org/10.1175/15200493(1959)087<0367:AOOAS>2.0.CO;2.

Darrigol, O., and U. Frisch, 2008: From Newton's mechanics to Euler's equations. Physica D, 237, 1855-1869, https://doi.org/ 10.1016/j.physd.2007.08.003.

Davis, C. A., and L. F. Bosart, 2003: Baroclinically induced tropical cyclogenesis. Mon. Wea. Rev., 131, 2730-2747, https://doi.org/ 10.1175/1520-0493(2003)131<2730:BITC>2.0.CO;2.

DeMaria, M., J. Kaplan, and J. Baik, 1993: Upper-level eddy angular momentum fluxes and tropical cyclone intensity change. J. Atmos. Sci., 50, 1133-1147, https://doi.org/10.1175/15200469(1993)050<1133:ULEAMF $>2.0 . C O ; 2$.

Ditchek, S. D., J. Molinari, and D. Vollaro, 2017: Tropical cyclone outflow-layer structure and balanced response to eddy forcings. J. Atmos. Sci., 74, 133-149, https://doi.org/10.1175/JASD-16-0117.1.

Doyle, J. D., and Coauthors, 2017: A view of tropical cyclones from above: The Tropical Cyclone Intensity (TCI) experiment. Bull. Amer. Meteor. Soc., 98, 2113-2134, https://doi.org/ 10.1175/BAMS-D-16-0055.1.

Dunion, J. P., 2011: Rewriting the climatology of the tropical North Atlantic and Caribbean Sea atmosphere. J. Climate, 24, 893908, https://doi.org/10.1175/2010JCLI3496.1.

Eastin, M. D., W. M. Gray, and P. G. Black, 2005: Buoyancy of convective vertical motions in the inner core of intense hurricanes. Part II: Case studies. Mon. Wea. Rev., 133, 209-227, https://doi.org/10.1175/MWR-2849.1.

Elsberry, R. L., and R. A. Jeffries, 1996: Vertical wind shear influences on tropical cyclone formation and intensification during TCM-92 and TCM-93. Mon. Wea. Rev., 124, 1374-1387, https://doi.org/10.1175/1520-0493(1996)124<1374: VWSIOT $>2.0 . \mathrm{CO} ; 2$.

Emanuel, K., 2012: Self-stratification of tropical cyclone outflow. Part II: Implications for storm intensification. J. Atmos. Sci., 69, 988-996, https://doi.org/10.1175/JAS-D-11-0177.1.

__, and R. Rotunno, 2011: Self-stratification of tropical cyclone outflow. Part I: Implications for storm structure. J. Atmos. Sci., 68, 2236-2249, https://doi.org/10.1175/JASD-10-05024.1.

Finocchio, P. M., S. J. Majumdar, D. S. Nolan, and M. Iskandarani, 2016: Idealized tropical cyclone responses to the height and depth of environmental wind shear. Mon. Wea. Rev., 144, 2155-2175, https://doi.org/10.1175/MWR-D-15-0320.1.

Fritsch, J. M., and R. A. Maddox, 1981: Convectively driven mesoscale weather systems aloft. Part I: Observations. J. Appl. Meteor. Climatol., 20, 910-921, https://doi.org/10.1175/15200450(1981)020<0910:PNTOTM > 2.0.CO;2. 
Ge, X., T. Li, and M. Peng, 2013: Effects of vertical shears and midlevel dry air on tropical cyclone developments. J. Atmos. Sci., 70, 3859-3875, https://doi.org/10.1175/JAS-D-13-066.1.

Grasso, L. D., M. Sengupta, J. F. Dostalek, R. Brummer, and M. DeMaria, 2008: Synthetic satellite imagery for current and future environmental satellites. Int. J. Remote Sens., 29, 4373 4384, https://doi.org/10.1080/01431160801891820.

Hanley, D., J. Molinari, and D. Keyser, 2001: A composite study of the interactions between tropical cyclones and upper-tropospheric troughs. Mon. Wea. Rev., 129, 2570-2584, https://doi.org/10.1175/ 1520-0493(2001)129<2570:ACSOTI >2.0.CO;2.

Holton, J. R., 1992: An Introduction to Dynamic Meteorology. Academic Press, 511 pp.

Jin, Y., and Coauthors, 2014: The impact of ice phase cloud parameterizations on tropical cyclone prediction. Mon. Wea. Rev., 142, 606-625, https://doi.org/10.1175/MWR-D13-00058.1.

Jones, S. C., 1995: The evolution of vortices in vertical shear. Part I: Initially barotropic vortices. Quart. J. Roy. Meteor. Soc., 121, 821-851, https://doi.org/10.1002/qj.49712152406.

Jordan, E. S., 1952: An observational study of the upper windcirculation around tropical cyclones. J. Meteor., 9, 340-346, https://doi.org/10.1175/1520-0469(1952)009<0340:AOSOTU> 2.0.CO;2.

Kaplan, J., M. DeMaria, and J. A. Knaff, 2010: A revised tropical cyclone rapid intensification index for the Atlantic and eastern North Pacific basins. Wea. Forecasting, 25, 220-241, https:// doi.org/10.1175/2009WAF2222280.1.

Knaff, J. A., C. R. Sampson, and K. D. Musgrave, 2018: An operational rapid intensification prediction aid for the western North Pacific. Wea. Forecasting, 33, 799-811, https://doi.org/ 10.1175/WAF-D-18-0012.1.

Komaromi, W. A., and J. D. Doyle, 2018: On the dynamics of tropical cyclone and trough interactions. J. Atmos. Sci., 75, 2687-2709, https://doi.org/10.1175/JAS-D-17-0272.1.

Marks, F. D., R. A. Houze, and J. F. Gamache, 1992: Dualaircraft investigation of the inner core of Hurricane Norbert. Part I: Kinematic structure. J. Atmos. Sci., 49, 919-942, https:// doi.org/10.1175/1520-0469(1992)049<0919:DAIOTI>2.0.CO;2.

McTaggart-Cowan, R., T. J. Galarneau, L. F. Bosart, R. W. Moore, and O. Martius, 2013: A global climatology of baroclinically influenced tropical cyclogenesis. Mon. Wea. Rev., 141, 1963-1989, https://doi.org/10.1175/MWR-D-1200186.1.

Merrill, R. T., 1988a: Characteristics of the upper-tropospheric environmental flow around hurricanes. J. Atmos. Sci., 45 1665-1677, https://doi.org/10.1175/1520-0469(1988)045<1665: COTUTE $>2.0 . \mathrm{CO} ; 2$.

, 1988b: Environmental influences on hurricane intensification. J. Atmos. Sci., 45, 1678-1687, https://doi.org/10.1175/ 1520-0469(1988)045<1678:EIOHI>2.0.CO;2.

_ , and C. S. Velden, 1996: A three-dimensional analysis of the outflow layer of Supertyphoon Flo (1990). Mon. Wea. Rev., 124, 47-63, https://doi.org/10.1175/1520-0493(1996)124<0047: ATDAOT $>2.0 . \mathrm{CO} ; 2$

Molinari, J., and D. Vollaro, 1989: External influences on hurricane intensity. Part I: Outflow layer eddy angular momentum fluxes. J. Atmos. Sci., 46, 1093-1105, https://doi.org/10.1175/ 1520-0469(1989)046<1093:EIOHIP>2.0.CO;2.

, and _ 1990: External influences on hurricane intensity. Part II: Vertical structure and response of the hurricane vortex. J. Atmos. Sci., 47, 1902-1918, https://doi.org/10.1175/15200469(1990)047<1902:EIOHIP>2.0.CO;2.
— S. Skubis, and D. Vollaro, 1995: External influences on hurricane intensity. Part III: Potential vorticity structure. J. Atmos. Sci., 52, 3593-3606, https://doi.org/10.1175/15200469(1995)052<3593:EIOHIP > 2.0.CO;2.

Möller, J. D., and L. J. Shapiro, 2002: Balanced contributions to the intensification of Hurricane Opal as diagnosed from a GFDL model forecast. Mon. Wea. Rev., 130, 1866-1881, https://doi.org/ 10.1175/1520-0493(2002)130<1866:BCTTIO >2.0.CO;2.

Montgomery, M. T., V. S. Nguyen, J. Persing, and R. K. Smith, 2009: Do tropical cyclones intensify by WISHE? Quart. J. Roy. Meteor. Soc., 135, 1697-1714, https://doi.org/10.1002/qj.459.

Onderlinde, M. J., and D. Nolan, 2016: Tropical cyclone-relative environmental helicity and the pathways to intensification in shear. J. Atmos. Sci., 73, 869-890, https://doi.org/10.1175/JASD-15-0261.1.

Ooyama, K. V., 1982: Conceptual evolution of the theory and modeling of the tropical cyclone. J. Meteor. Soc. Japan, 60 , 369-380, https://doi.org/10.2151/jmsj1965.60.1_369.

Palmén, E., and H. Riehl, 1957: Budget of angular momentum and energy in tropical cyclones. J. Meteor., 14, 150-159, https://doi.org/ 10.1175/1520-0469(1957)014<0150:BOAMAE $>2.0 . \mathrm{CO} ; 2$.

Persing, J., M. T. Montgomery, and R. E. Tuleya, 2002: Environmental interactions in the GFDL hurricane model for Hurricane Opal. Mon. Wea. Rev., 130, 298-317, https://doi.org/ 10.1175/1520-0493(2002)130<0298:EIITGH > 2.0.CO;2.

Rappin, E. D., and D. S. Nolan, 2012: The effect of vertical shear orientation on tropical cyclogenesis. Quart. J. Roy. Meteor. Soc., 138, 1035-1054, https://doi.org/10.1002/qj.977.

_ - M. C. Morga, and G. J. Tripoli, 2011: The impact of outflow environment on tropical cyclone intensification and structure. J. Atmos. Sci., 68, 177-194, https://doi.org/10.1175/2009JAS2970.1.

Reasor, P. D., and M. T. Montgomery, 2015: Evaluation of a heuristic model for tropical cyclone resilience. J. Atmos. Sci., 72, 1765-1782, https://doi.org/10.1175/JAS-D-14-0318.1.

,-- , and L. D. Grasso, 2004: A new look at the problem of tropical cyclones in vertical shear flow: Vortex resiliency. J. Atmos. Sci., 61, 3-22, https://doi.org/10.1175/1520-0469(2004) 061<0003:ANLATP $>2.0$. CO;2.

Riemer, M., and F. Laliberté, 2015: Secondary circulation of tropical cyclones in vertical wind shear: Lagrangian diagnostic and pathways of environmental interaction. J. Atmos. Sci., 72, 3517-3536, https://doi.org/10.1175/JAS-D-14-0350.1.

—, M. T. Montgomery, and M. E. Nicholls, 2010: A new paradigm for intensity modification of tropical cyclones: Thermodynamic impact of vertical wind shear on the inflow layer. Atmos. Chem. Phys., 10, 3163-3188, https://doi.org/10.5194/ acp-10-3163-2010.

Rotunno, R., and K. A. Emanuel, 1987: An air-sea interaction theory for tropical cyclones. Part II: Evolutionary study using a nonhydrostatic axisymmetric numerical model. J. Atmos. Sci., 44, 542-561, https://doi.org/10.1175/1520-0469(1987) $044<0542$ :AAITFT $>2.0$. CO; 2 .

Rozoff, C. M., C. S. Velden, J. Kaplan, J. P. Kossin, and A. J. Wimmers, 2015: Improvements in the probabilistic prediction of tropical cyclone rapid intensification with passive microwave observations. Wea. Forecasting, 30, 1016-1038, https:// doi.org/10.1175/WAF-D-14-00109.1.

Ryglicki, D. R., and R. E. Hart, 2015: An investigation of centerfinding techniques for tropical cyclones in mesoscale models. J. Appl. Meteor. Climatol., 54, 825-846, https://doi.org/10.1175/ JAMC-D-14-0106.1.

J. H. Cossuth, D. H. Hodyss, and J. D. Doyle, 2018a: The unexpected rapid intensification of tropical cyclones in moderate 
vertical wind shear. Part I: Overview and observations. Mon. Wea. Rev., 146, 3773-3800, https://doi.org/10.1175/MWRD-18-0020.1.

_ J. D. Doyle, Y. Jin, D. Hodyss, and J. H. Cossuth, 2018b: The unexpected rapid intensification of tropical cyclones in moderate vertical wind shear. Part II: Vortex tilt. Mon. Wea. Rev., 146, 3801-3825, https://doi.org/10.1175/MWR-D-18-0021.1.

Schecter, D. A., M. T. Montgomery, and P. D. Reasor, 2002: A theory for the vertical alignment of a quasigeostrophic vortex. J. Atmos. Sci., 59, 150-168, https://doi.org/10.1175/15200469(2002)059<0150:ATFTVA > 2.0.CO;2.

Schmidt, J. M., and W. R. Cotton, 1990: Interactions between upper and lower tropospheric gravity waves on squall line structure and maintenance. J. Atmos. Sci., 47, 1205-1222, https:// doi.org/10.1175/1520-0469(1990)047<1205:IBUALT>2.0.CO;2.

Sears, J., and C. S. Velden, 2014: Investigating the role of the upper-levels in tropical cyclone genesis. Trop. Cyclone Res. Rev., 3, 91-110, https://doi.org/10.6057/2014TCRR02.03.

Shapiro, L. J., and J. D. Möller, 2003: Influence of atmospheric asymmetries on the intensification of Hurricane Opal: Piecewise PV inversion diagnosis of a GFDL model forecast. Mon. Wea. Rev., 131, 1637-1649, https://doi.org/10.1175//2552.1.

Shi, J. J., S. Chang, and S. Raman, 1997: Interaction between Hurricane Florence (1988) and an upper-tropospheric westerly trough. J. Atmos. Sci., 54, 1231-1247, https://doi.org/ 10.1175/1520-0469(1997)054<1231:IBHFAA > 2.0.CO;2.

Stewart, S. R., 2017: National Hurricane Center Tropical Cyclone Report: Hurricane Matthew (28 September-9 October 2016). Rep. AL142016, NWS TPC/National Hurricane Center, 96 pp., https://www.nhc.noaa.gov/data/tcr/AL142016_Matthew.pdf.

Tang, B., and K. Emanuel, 2010: Midlevel ventilation's constraint on tropical cyclone intensity. J. Atmos. Sci., 67, 1817-1830, https://doi.org/10.1175/2010JAS3318.1.
— , and 2012: Sensitivity of tropical cyclone intensity to ventilation in an axisymmetric model. J. Atmos. Sci., 69, 2394 2413, https://doi.org/10.1175/JAS-D-11-0232.1.

Trier, S. B., and R. D. Sharman, 2009: Convection-permitting simulations of the environment supporting widespread turbulence within the upper-level outflow of a mesoscale convective system. Mon. Wea. Rev., 137, 1972-1990, https://doi.org/ 10.1175/2008MWR2770.1.

Van Delst, P., 2013: CRTM: v2.1.3 User's Guide. Joint Center for Satellite Data Assimilation, $232 \mathrm{pp}$.

Velden, C. S., and J. Sears, 2014: Computing deep-tropospheric vertical wind shear analyses for tropical cyclone applications: Does the methodology matter? Wea. Forecasting, 29, 11691180, https://doi.org/10.1175/WAF-D-13-00147.1.

— C. M. Hayden, S. J. Nieman, W. P. Menzel, S. Wanzong, and J. S. Goerss, 1997: Upper-tropospheric winds derived from geostationary satellite water vapor observations. Bull. Amer. Meteor. Soc., 78, 173-195, https://doi.org/10.1175/15200477(1997)078<0173:UTWDFG > 2.0.CO;2.

Wang, Y., and C.-C. Wu, 2004: Current understanding of tropical cyclone structure and intensity changes-A review. Meteor. Atmos. Phys., 87, 257-278, https://doi.org/10.1007/s00703003-0055-6.

Wu, C.-C., and K. Emanuel, 1993: Interaction of a baroclinic vortex with background shear: Application to hurricane movement. J. Atmos. Sci., 50, 62-76, https://doi.org/10.1175/ 1520-0469(1993)050<0062:IOABVW >2.0.CO;2.

— Sci., 51, 1995-2003, https://doi.org/10.1175/1520-0469(1994) 051<1995:OHOS $>2.0 . \mathrm{CO} ; 2$.

Zhang, F., and D. Tao, 2013: Effects of vertical wind shear on the predictability of tropical cyclones. J. Atmos. Sci., 70, 975-983, https://doi.org/10.1175/JAS-D-12-0133.1. 